\title{
CPAMD8 loss-of-function underlies non-dominant congenital glaucoma with variable anterior segment dysgenesis and abnormal extracellular matrix
}

\author{
Juan-Manuel Bonet-Fernández ${ }^{1,2,3} \cdot$ José-Daniel Aroca-Aguilar ${ }^{1,2,3} \cdot$ Marta Corton $^{4,5} \cdot$ Ana-Isabel Ramírez ${ }^{3,6}$. \\ Susana Alexandre-Moreno ${ }^{1,2,3} \cdot$ María-Teresa García-Antón $^{6}$. Juan-José Salazar³,6 • Jesús-José Ferre-Fernández ${ }^{1,2,3}$. \\ Raquel Atienzar-Aroca ${ }^{1,2,3}$. Cristina Villaverde ${ }^{4,5}$. Ionut lancu ${ }^{4,5}$. Alejandra Tamayo ${ }^{4}$. \\ Carmen-Dora Méndez-Hernández ${ }^{3,7,8}$. Laura Morales-Fernández ${ }^{3,7,8}$. Blanca Rojas 3,6,9 $^{3,6}$ Carmen Ayuso 4,5 . \\ Miguel Coca-Prados ${ }^{10} \cdot$ José-Maria Martinez-de-la-Casa ${ }^{3,7,8} \cdot$ Julián García-Feijoo ${ }^{3,7,8} \cdot$ Julio Escribano ${ }^{1,2,3}$ (1)
}

Received: 27 January 2020 / Accepted: 3 April 2020

(c) Springer-Verlag GmbH Germany, part of Springer Nature 2020

\begin{abstract}
Abnormal development of the ocular anterior segment may lead to a spectrum of clinical phenotypes ranging from primary congenital glaucoma (PCG) to variable anterior segment dysgenesis (ASD). The main objective of this study was to identify the genetic alterations underlying recessive congenital glaucoma with ASD (CG-ASD). Next-generation DNA sequencing identified rare biallelic CPAMD8 variants in four patients with CG-ASD and in one case with PCG. CPAMD8 is a gene of unknown function and recently associated with ASD. Bioinformatic and in vitro functional evaluation of the variants using quantitative reverse transcription PCR and minigene analysis supported a loss-of-function pathogenic mechanism. Optical and electron microscopy of the trabeculectomy specimen from one of the CG-ASD cases revealed an abnormal anterior chamber angle, with altered extracellular matrix, and apoptotic trabecular meshwork cells. The CPAMD8 protein was immunodetected in adult human ocular fluids and anterior segment tissues involved in glaucoma and ASD (i.e., aqueous humor, non-pigmented ciliary epithelium, and iris muscles), as well as in periocular mesenchyme-like cells of zebrafish embryos. CRISPR/Cas9 disruption of this gene in F0 zebrafish embryos (96 hpf) resulted in varying degrees of gross developmental abnormalities, including microphthalmia, pharyngeal maldevelopment, and pericardial and periocular edemas. Optical and electron microscopy examination of these embryos showed iridocorneal angle hypoplasia (characterized by altered iris stroma cells, reduced anterior chamber, and collagen disorganized corneal stroma extracellular matrix), recapitulating some patients' features. Our data support the notion that CPAMD 8 loss-of-function underlies a spectrum of recessive CG-ASD phenotypes associated with extracellular matrix disorganization and provide new insights into the normal and disease roles of this gene.
\end{abstract}

\section{Introduction}

Primary congenital glaucoma (PCG) and anterior segment dysgenesis (ASD) are related and heterogeneous diseases that may severely impair visual function. They result from

Juan-Manuel Bonet-Fernández and José-Daniel Aroca-Aguilar contributed equally to this work.

Electronic supplementary material The online version of this article (https://doi.org/10.1007/s00439-020-02164-0) contains supplementary material, which is available to authorized users.

Julio Escribano

julio.escribano@uclm.es

Extended author information available on the last page of the article diverse developmental abnormalities of the ocular anterior segment structures, which include the Schlemm's canal (SC), trabecular meshwork (TM), ciliary body (CB), iris, and cornea (Kupfer and Kaiser-Kupfer 1978, 1979). PCG is caused by a malformation restricted to the TM and/or SC, i.e., trabeculodysgenesis or gonyodysgenesis (Gould and John 2002), which results in elevated intraocular pressure (IOP), and in the classical triad of epiphora, blepharospasm, and photophobia (Papadopoulos et al. 2013). Ocular enlargement and curvilinear breaks in the Descemet's membrane (Haab striae) may also be present in some patients. Typical PCG is usually diagnosed in the neonatal or infantile period. On the other hand, ASD malformation and its associated clinical features are highly variable and may include underdevelopment of the iris (iris hypoplasia), altered corneal 
diameter, corneal opacity (leukoma), and vascularization, posterior embryotoxon (thickened and displaced Schwalbe's line), displacement of the pupil (corectopia), the existence of more than one pupillary opening (polycoria), an abnormal iridocorneal angle, anterior synechiae between the iris and posterior corneal surface, displacement of the lens (ectopia lentis), and adhesion between the cornea and the lens or the pupillary border (Peters' anomaly) (Sowden 2007). The specific combination of posterior embryotoxon, iris hypoplasia, corectopia/polycoria, and/or anterior synechiae is recognized as a separate diagnostic entity called AxenfeldRieger anomaly, which combines with systemic anomalies in the Axenfeld-Rieger syndrome (Reis and Semina 2011). In addition, ASD is generally associated with an approximate $50 \%$ risk for glaucoma (Reis and Semina 2011).

Both PCG and ASD are believed to be caused by defective patterning, migration, and/or differentiation of periocular mesenchyme cells, which derive mainly from the neural crest but also from paraxial mesoderm (Creuzet et al. 2005). Moreover, PCG and ASD may be caused by the same genes (Gould and John 2002). In this line, mutations in CYP1B1 mainly produce PCG but have also been detected in some patients with Peters' (Vincent et al. 2001, 2006) and Rieger's anomalies (Chavarria-Soley et al. 2006). A common feature of many ASD diseases is disturbance of the extracellular matrix (ECM) composition and abundance, although the specific underlying alterations remain mostly unidentified. ECM signaling has been postulated as being one relevant component of the networks involved in anterior segment development (Gould et al. 2004). Functional disruption of this complex developmental regulation may occur at different points in different patients, leading to genetic and clinical variability, and to the overlapping phenotypic features observed in different ASD subtypes. This scenario presents challenges in terms of the correct diagnosis and classification of patients.

Unlike other ASDs, which are frequently dominant, PCG is generally transmitted in an autosomal-recessive fashion, but incomplete penetrance and variable expressivity are present in all these dysgenesis (Sarfarazi and Stoilov 2000). The first identified and most prevalent cause of recessive PCG is CYP1B1 loss-of-function (LoF) (Sarfarazi and Stoilov 2000; Stoilov et al. 1997), which is present in $18-48 \%$ of nonconsanguineous European patients (Campos-Mollo et al. 2009; Colomb et al. 2003; López-Garrido et al. 2013; Weisschuh et al. 2009). Additional genes reported to play a role in this pathology are LTBP2 (Ali et al. 2009), MYOC (Kaur et al. 2005), FOXC1 (Medina-Trillo et al. 2015, 2016), TEK (Souma et al. 2016), and GPATCH3 (Ferre-Fernández et al. 2017), illustrating the genetic heterogeneity of this disease.

The principal known genes that cause different types of typical dominant ASD are FOXC1 (Nishimura et al. 1998) and PITX2 (Semina et al. 1996), which are mainly involved in Axenfeld-Rieger alterations. In addition, $P A X 6$ is linked to Peters' anomaly (Hanson et al. 1994) and aniridia (Jordan et al. 1992). Cases of recessive ASD transmission have also been described (Reis and Semina 2011). Recently, in three families, rare CPAMD 8 variants have been identified as the cause of an atypical form of autosomal recessive ASD characterized by bilateral iris hypoplasia, ectopia lentis, corectopia, ectropion uveae, and cataracts (ASD8, OMIM \# 617319) (Cheong et al. 2016).

In this study we investigate the genetic alterations underlying glaucoma with ASD (CG-ASD) and isolated PCG in a group of unrelated patients who have no family history of the disease and who have variable clinical features. We found that these patients carry rare biallelic CPAMD $8 \mathrm{LoF}$ variants associated with ECM alterations. In addition, we show that this gene plays a role in ocular ECM formation and in early ocular development, providing new insights into the molecular pathways involved in anterior segment morphogenesis and associated diseases.

\section{Methods}

\section{Subjects}

Patients were recruited at San Carlos Clinic Hospital and University Hospital Fundación Jimenez Diaz, Madrid, Spain. Four patients diagnosed with CG-ASD and 50 PCG patients selected from a cohort composed of 150 unrelated cases (López-Garrido et al. 2013) with no family history of the disease and no CYP1B1, FOXC1, FOXC2 or MYOC pathogenic variants were selected for genetic studies. The clinical features of this PCG cohort have previously been reported (Campos-Mollo et al. 2009; López-Garrido et al. 2013). Glaucoma specialists carried out the clinical examination of all patients, which included slit-lamp biomicroscopy, gonioscopy, biometry, IOP measurement, and ophthalmoscopy. PCG diagnosis was performed as previously described (López-Garrido et al. 2013). The human study and informed consent procedures were approved by the Ethics Committee for Human Research of the University Hospital Fundación Jiménez Díaz and Hospital Clínico San Carlos (approval number 13/388-E). The research followed the tenets of the Declaration of Helsinki. Informed written consents were obtained prior to participants' inclusion in the study.

\section{Human tissue samples}

A human eye from a 45-year-old Caucasian female donor (cadaver) with no reported ocular pathology was obtained within $24 \mathrm{~h}$ after enucleation from the USA National Disease Research Interchange. Tears collected from one healthy 32-year-old female volunteer, a member of the research 
group, were used for Western blot analysis. Tissue processing is described in the Suppl. Methods.

\section{Animals}

Wild-type AB zebrafish (Danio rerio) were maintained at $28{ }^{\circ} \mathrm{C}$ with a $14 \mathrm{~h}$ on/10 h off light cycle and were fed a standard diet according to established protocols (Westerfield 2000). Detailed embryo management protocols are available in the Suppl. Methods. All animal husbandry and experiments were approved and conducted in accordance with the guidelines set forth by the Institutional Animal Research Committee of the University of Castilla-La Mancha (approval number PR-2015-04-10).

\section{Next generation sequencing}

Genomic DNA was extracted from the subjects' peripheral blood, using the QIAamp DNA Blood Mini Kit (Quiagen) and processed for targeted NGS using a custom gene panel composed of coding and non-coding exons and $25 \mathrm{pb}$ of intronic boundaries of 82 genes involved in glaucoma, ASD, and ocular development. The gene panel also included full intronic sequences and 22 putative regulatory regions of CYP1B1 and LRP2 genes. The panel was developed using the SureSelect QXT capture technology (Agilent Technologies). Detailed protocols are available in the Suppl. Methods. Bioinformatic analysis was performed using standard procedures and custom in-house pipelines for both SNVs analysis and read-count based CNVs analysis, as previously reported (Ceroni et al. 2019) and are described in detail in the Suppl. Methods.

\section{Variant prioritization and validation}

Candidate disease-causing variants were identified through the application of a multistep filtering approach. Initially, common variants, defined as those with a minor allele frequency higher than $1 \%$ in the Exome Aggregation Consortium (ExAC) (https://exac.broadinstitute.org/) or gnomAD (https://gnomad.broadinstitute.org/) browsers and with a genotype quality lower than 50 reads were filtered out. Next, LoF variants (nonsense, indels producing a frameshift and variants affecting canonical splicing sites) were selected. If no candidate variants met the later stringent functional criterium, we included missense, synonymous, and noncanonical splicing variants bioinformatically predicted to cause deleterious effects, using the software described below. Finally, to identify potential recessive genotypes, we selected variants in compound heterozygosis or homozygosis. The detailed variant filtering pipelines followed in this study are summarized in Figs. 1a and 3a. The DNA sample for WES was processed as previously described
(Ferre-Fernández et al. 2017). Candidate variants identified by NGS were verified and segregated in the corresponding families by Sanger sequencing following PCR amplification using conditions and primers indicated Table S1.

\section{CNV validation}

A CNV affecting the CPAMD8 exon 23 was validated and segregated in the family using copy-number quantification by droplet digital PCR (ddPCR). Detailed protocols are available in the Suppl. Methods.

\section{Quantitative reverse transcription PCR (qRT-PCR)}

Density gradient centrifugation of peripheral whole blood using Ficoll-Paque PLUS (GE Healthcare Biosciences) was used to isolate mononuclear cells from CG-ASD-104 family members and from two healthy controls who did not carry pathogenic CPAMD8 variants. RNA was isolated using the RNeasy Minikit (Qiagen \#74104) and treated with RNasefree DNase I according to the manufacturer's instructions. Purified RNA was used for cDNA synthesis using RevertAid First-Strand cDNA Synthesis Kits (Thermo Scientific \#K1622). The expression of CPAMD8 mRNA relative to $P R L P 0$ mRNA was determined using the $2^{-\Delta \Delta C t}$ method (Livak and Schmittgen 2001). Detailed protocols are available in the Suppl. Methods.

\section{Splicing analysis}

Both wild-type and mutant exon 15 of CPAMD8 and their flanking sequences (390 bp and $280 \mathrm{bp}$ of introns 14 and 15 , respectively) were amplified from the genomic DNA of patient CG-ASG-160. Similarly, CPAMD8 wild-type and mutant genomic fragments encompassing exons 33-34 and the corresponding flanking sequences (final $173 \mathrm{bp}$ of intron 32 and initial 383 bp of intron 34) were obtained from the ASG-CG-30 proband. Detailed amplification protocols are provided in the Suppl. Methods. PCR products were cloned into the pTBN1 plasmid (a gift from Franco Pagani, Addgene plasmid \#15125). Minigene DNA constructs were transfected into HEK-293T cells; the transcripts were analyzed by RT-PCR and direct Sanger sequencing. In vivo splicing of CPAMD8 was analysed using cDNA obtained from one of the healthy controls described above. Detailed protocols are available in the Suppl. Methods.

\section{Western blotting and antibodies}

Samples used for Western blot analyses were fractionated by SDS-PAGE using the Mini-PROTEAN III Gel Electrophoresis System and transferred onto Hybond ECL nitrocellulose membranes (Amersham) for immunodetection 


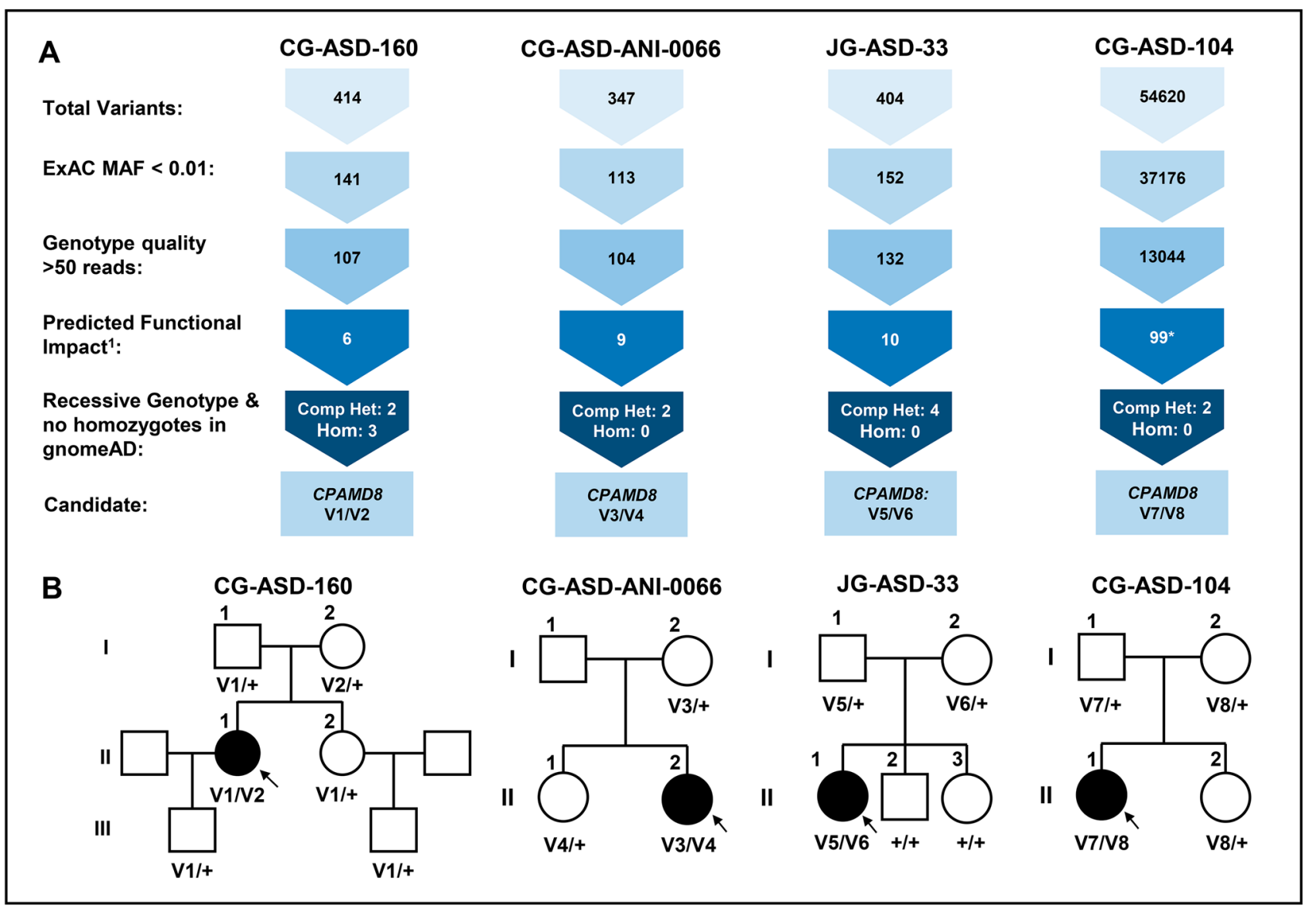

Fig. 1 Variant filtering scheme used for candidate variant identification and pedigree analysis of CPAMD 8 variants in patients with congenital glaucoma and anterior segment dysgenesis. a Gene variants were identified by NGS using a gene panel comprising 121 genes (CG-ASD-160, CG-ASD-ANI-0066 and JG-ASD-33) or by WES (CG-ASD-104) and filtered as indicated to identify candidate variants. b Candidate variant segregation. The black symbol indicates the presence of the disease. Arrows in the pedigrees show the index case. + : wild-type allele. ${ }^{1}$ Frameshift, stop gained, start lost, splicing acceptor, and missense variants. V1: p.(Arg1231Profs*64); V2: c.1758+1_1758+4del; V3: delex23; V4: p.(Ala688Asp)c.2243+4A>C; V5: p.(Pro936Leu); V6: p.(Ala1331Ser); V7: p.(Lys845fs*13); V8: p.(Arg668*)

their histology. After uranyl acetate and lead citrate contrast, sections were photographed using a transmission-electron microscope (JEOL JEM 1010). Detailed protocols are available in the Suppl. Methods.

\section{Fluorescence immunohistochemistry (FIHC)}

Paraffin-embedded human eye sections $(3 \mu \mathrm{m})$ and zebrafish embryo cryosections $(10-14 \mu \mathrm{m})$ were incubated with a rabbit anti-human CPAMD8 1:200 primary antibody (HPA031328, Sigma) and Cy2 donkey anti-rabbit 1:1000 secondary antibody (Jackson ImmunoResearch). Sections were also counterstained with DAPI and visualized using an LSM710 Zeiss confocal microscope. Fluorescence emitted by DAPI, the Cy2-conjugated antibody, and embryo autofluorescence were registered at $411-464 \mathrm{~nm}, 490-518 \mathrm{~nm}$, 
and 553-677 $\mathrm{nm}$ respectively. Detailed protocols are available in the Suppl. Methods.

\section{Fluorescent whole mount immunohistochemistry (FWIHC)}

Ninety-six hpf phenylthiourea-treated and fixed embryos were incubated with a rabbit anti-human CPAMD8 1:200 primary antibody (HPA031328, Sigma) and a Cy2 donkey anti-rabbit 1:1500 secondary antibody (Jackson ImmunoResearch). Embryos were also counterstained with DAPI, mounted in low-melting agarose, and visualized in an LSM710 Zeiss confocal microscope. Fluorescence was detected as described earlier. Z-Stack maximum intensity projections of embryos were obtained with ZEN software (Zeiss). Detailed protocols are available in the Suppl. Methods.

\section{CRISPR/Cas 9 gene editing}

Deletions of approximately $100 \mathrm{bp}$ were generated either in exon 4 or in exon 25 using the crRNAs pairs described in the Suppl. Methods. Cas9/tracrRNA/crRNA complex (approximately $3 \mathrm{nl}$ ) was injected into the animal pole of one-cell stage embryos (50-250 embryos/experiment) using a Femtojet 5247 microinjector (Eppendorf) under a Nikon DS-Ri2 stereomicroscope. As a negative control, embryos were injected with Cas9/tracrRNA and no crRNA. For each crRNA pair and control, at least three independent experiments were performed using different zebrafish progenitors in each experiment. Detailed protocols are available in the Suppl. Methods.

\section{In silico analyses}

The different programs used to evaluate the deleterious effect of the different variants, to identify protein domains, and to align aa sequences are described in the Suppl. Methdos. Novel variants were named using directions from Mutalyzer (https://mutalyzer.nl/), according to reference sequences NM_015692.4 and NP_056507.3.

\section{Statistics}

Statistical comparisons between groups were performed using either the $t$-test or the one-way ANOVA. Statistical significance of the difference in the proportion of rare SNVs between PCG patients and the gnomAD database was determined by the chi-square test. Statistical analysis of the data was performed using the SigmaStat 2.0 software (SPSS Science Inc.).

\section{Results}

We investigated by next generation sequencing (NGS) the underlying genetic alterations in an atypical group of four glaucoma patients with variable ASD who, in addition, did not present with a family history of the disease. Gene variants were screened in three patients using an in-house developed gene panel comprising 82 genes related to ASD, glaucoma or ocular development. The fourth case was analyzed by trio whole-exome sequencing (WES). The variant filtering algorithm aimed to identify rare variants with potential recessive genotypes (i.e., a homozygous or compound heterozygous state) and predicted high functional impact (frameshift, non-sense, missense, and donor/acceptor splicing sites). The filtering pipeline revealed biallelic CPAMD8 variants in the first three patients (CG-ASD-160, ASD-ANI-0066, and JG-ASD-33, Fig. 1a). CPAMD8 is a gene recently implicated in atypical autosomal recessive ASD (Cheong et al. 2016). One allele identified in patient CG-ASD-160 was a single-nucleotide insertion (c.3686_3687insT) predicted to cause a frameshift and a premature termination codon (PTC) in the mutant protein (p.(Arg1231Profs*64)), and it was positioned within a conserved alpha-2 macroglobulin (A2M) thiolester-containing domain (Fig. 2). The second allele (c.1758+1_1758+4del) deleted the canonical GT dinucleotide required for normal splicing. It was predicted in silico to break the donor site (Table 1), potentially leading to the retention of intron 15 in the processed mRNA, as well as to a PCT at the fifth codon in the mutant open reading frame (ORF). The former variant has not been reported in the gnomAD database and the latter has a very low frequency $(9.398 \mathrm{e}-5)$, with no homozygotes described (Table 1). Sanger sequencing confirmed the presence of these variants in the patient (Fig. S1) and in five family members, showing an inheritance pattern compatible with recessive transmission (Fig. 1b). The filtering pipeline identified in the second patient (CG-ASD-ANI-0066) two close nucleotide substitutions: c.2063C $>$ A and c.2070+4A $>$ C (Fig. 1a). Visual inspection of aligned read sequences indicated that both variants could be in the same chromosome. The former variant was predicted to result in the amino acid change p.(Ala688Asp) in the bait region domain of the protein (Fig. 2a), while the later affected the intron 17 donor splicing site (Fig. 2b). None of these variants have previously been reported in gnomAD. Bioinformatic pathogenic predictions classified the first variant as tolerated or probably damaging, whilst two different algorithms predicted the second one would break the intron 17 donor site (Table 1), potentially resulting in the retention of this intron in the mature mRNA with the subsequent creation of a new ORF that leads to creation of a PTC in the mutant 


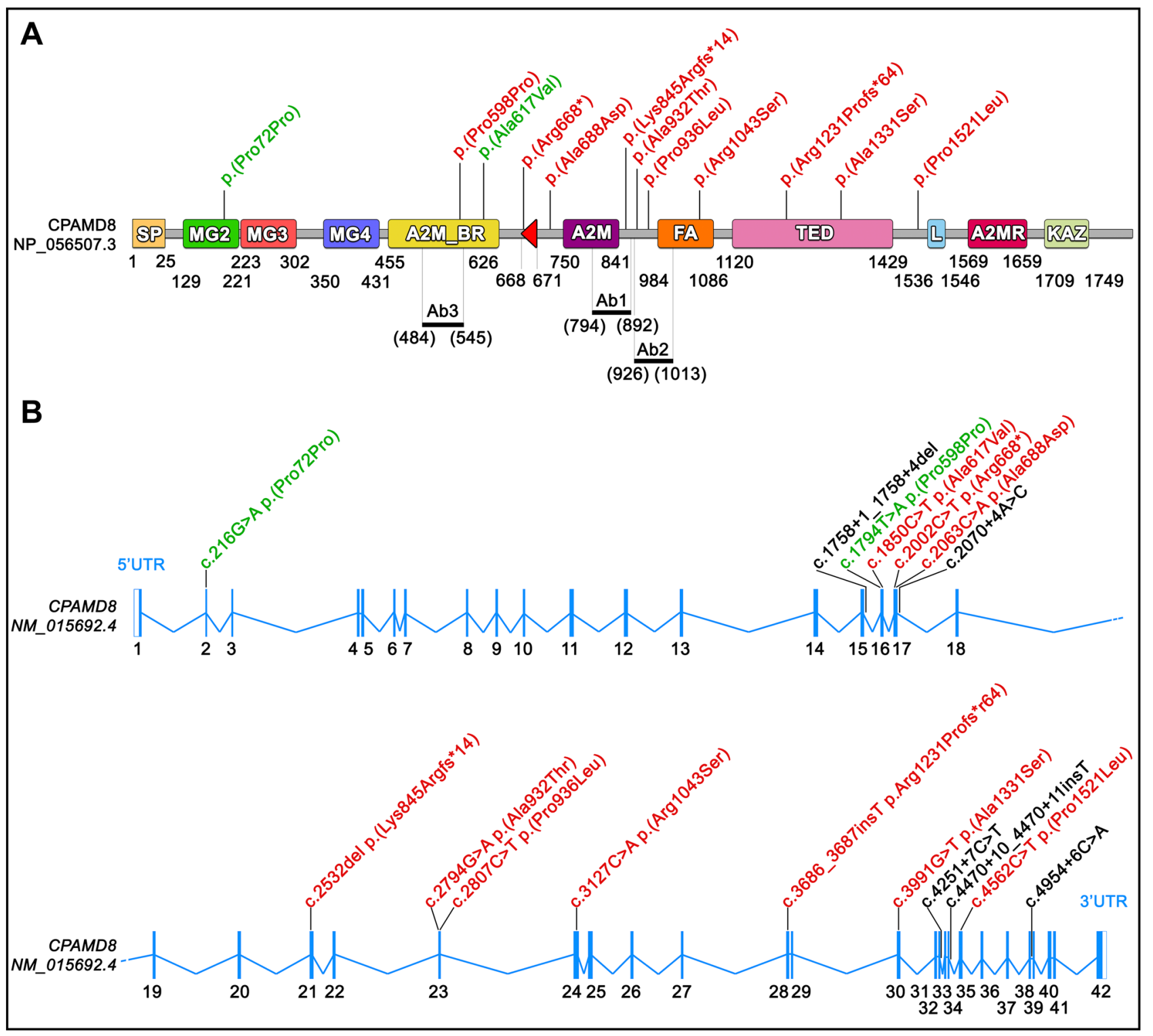

Fig. 2 Localization of CPAMD8 variants identified in this study. a Scheme of the CPAMD8 protein domain organization indicating the localization of coding variants (positions are referred to sequence NP_056507). Domains are indicated according to the Pfam database (https://pfam.xfam.org/). Ab1, Ab2, and Ab3: epitopes recognized by the three antibodies used in this study. Red triangle: position of the four Arg residues of the cleavage motif. A2M: Alpha-2-macroglobulin domain. A2M_BRD: Alpha-2-macroglobulin bait region domain. A2MR: Alpha-macroglobulin receptor binding domain. FA:

mRNA (p.(Glu691Valfs*99)). Segregation analysis confirmed the presence in cis of both heterozygous nucleotide substitutions in the patient and in her mother, whereas the patient's sister inherited the wild-type allele (Fig. 1b and Fig. S1). Further bioinformatic copy number variation $(\mathrm{CNV})$ analysis using a bioinformatic read-depth approach revealed a putative exon- 23 deletion, which was further
Farnesoic acid 0-methyl transferase domain. MG2: Macroglobulin 2 domain of Alpha2-Macroglobulin. MG3: Macroglobulin 3 domain of Alpha2-Macroglobulin. MG4: Macroglobulin 4 domain of Alpha2Macroglobulin. L: low complexity Pfam domain. KAZ: Kazal-type serine protease inhibitor domain. SP: signal peptide. TED: A-macroglobulin thioester domain. b Genomic localization of CPAMD8 variants identified in this study. Positions correspond to reference sequence NM_015692. Red: missense variants. Green: synonymous variants. Black: intronic variants

validated by droplet digital PCR (ddPCR) (Fig. S3); to the best of our knowledge, it has not previously been reported. Segregation by ddPCR assay showed that the unaffected proband's sister inherited the exon 23 deletion in the heterozygous state (Fig. S3). This variant was predicted to cause a frameshift at codon 979 introducing a PCT downstream from the deletion (p.(Ala979Argfs*49)). Although 
Table 1 Biallelic CPAMD8 variants identified in patients diagnosed with CG-ASD or PCG

\begin{tabular}{|c|c|c|c|c|c|c|c|}
\hline Family & $\begin{array}{l}\text { Chromo- } \\
\text { some position } \\
\text { (hgv37) }\end{array}$ & cDNA (NM_015692.4) & $\begin{array}{l}\text { Protein } \\
\text { (NP_056507.3) }\end{array}$ & $\begin{array}{l}\text { Allele } \\
\text { frequency/ } \\
\text { number of } \\
\text { homozygotes } \\
\text { (gnomAD) }\end{array}$ & ${ }^{\mathrm{a} S I F T} /{ }^{b} \mathrm{Polyphen} /{ }^{\mathrm{c}} \mathrm{EVEP} /{ }^{\mathrm{d}} \mathrm{DANN}$ & $\begin{array}{l}{ }^{\mathrm{e}} \mathrm{HSF} 3.0 \\
\text { predicted } \\
\text { effects (\# of } \\
\text { algorithms) }\end{array}$ & $\begin{array}{l}\text { Reference } \\
\text { number }\end{array}$ \\
\hline CG-ASG-160 & $\begin{array}{l}19: 17025566 \\
19: 17088173\end{array}$ & $\begin{array}{l}\text { c.3686_3687insT } \\
\text { c. } 1758+1 \_1758+4 \mathrm{del}\end{array}$ & $\begin{array}{l}\text { p.(Arg1231Profs*64) } \\
\text { f p.(Val587Serfs*5) }\end{array}$ & $\begin{array}{l}0(0) \\
9.398 \mathrm{e}-5(0)\end{array}$ & $\begin{array}{l}-1-1-1- \\
-1-1-1-\end{array}$ & $\overline{-}$ & $\begin{array}{l}\text { ss3943621968 } \\
\text { rs577740063 }\end{array}$ \\
\hline $\begin{array}{l}\text { CG-ASG- } \\
\text { ANI-0066 }\end{array}$ & $\begin{array}{c}19: 17085914 \\
19: 17085903 \\
19: 17049179- \\
17049281\end{array}$ & $\begin{array}{l}\text { c. } 2063 \mathrm{C}>\mathrm{A} \\
\text { c. } 2070+4 \mathrm{~A}>\mathrm{C} \\
\operatorname{del} \operatorname{ex} 23\end{array}$ & $\begin{array}{l}\text { p.Ala688Asp } \\
\text { f. } \text { p.(Glu691Valfs*99) } \\
\text { p.(Ala979Argfs*49) }\end{array}$ & $\begin{array}{l}0(0) \\
0(0) \\
0(0)\end{array}$ & $\begin{array}{l}\mathrm{T}(0.33) / \mathrm{PD}(1) /-/- \\
-/-/-/-\end{array}$ & $\begin{array}{l}- \\
\text { DSB (2) } \\
-\end{array}$ & $\begin{array}{l}\text { ss3943621972 } \\
\text { ss3943621971 }\end{array}$ \\
\hline J-ASD-33 & $\begin{array}{l}19: 17017798 \\
19: 17049243\end{array}$ & $\begin{array}{l}\text { c. } 3991 \mathrm{G}>\mathrm{T} \\
\text { c. } 2807 \mathrm{C}>\mathrm{T}\end{array}$ & $\begin{array}{l}\text { p.(Ala1331Ser) } \\
\text { p.(Pro936Leu) }\end{array}$ & $\begin{array}{l}7.657 \mathrm{e}-5(0) \\
0.0008707(0)\end{array}$ & $\begin{array}{l}\mathrm{T}(0.12) / \mathrm{PD}(0.624) / \mathrm{M} / 0.983 \\
\mathrm{~T}(0.1) / \mathrm{B}(0.17) / \mathrm{M} / 0.979\end{array}$ & $\begin{array}{l}\text { CSSA (1) } \\
\text { ESEB (5) } \\
\text { No effect }\end{array}$ & $\begin{array}{l}\text { rs } 754711230 \\
\text { rs200400599 }\end{array}$ \\
\hline CG-ASG-104 & $\begin{array}{l}19: 17058014 \\
19: 17085975\end{array}$ & $\begin{array}{l}\text { c. } 2532 \mathrm{del} \\
\text { c. } 2002 \mathrm{C}>\mathrm{T}\end{array}$ & $\begin{array}{l}\text { p.(Lys845Argfs*14) } \\
\text { p.(Arg668*) }\end{array}$ & $\begin{array}{l}4.02071 \mathrm{e}-06 \\
\quad(0) \\
5.481 \mathrm{e}-5(0)\end{array}$ & $\begin{array}{l}-I-I-I- \\
-1-I-I-\end{array}$ & $\begin{array}{l}- \\
-\end{array}$ & $\begin{array}{l}\text { ss } 3943621970 \\
\text { rs } 200025505\end{array}$ \\
\hline PCG-30 & $\begin{array}{l}19: 17086870 \\
19: 17014360\end{array}$ & $\begin{array}{l}\text { c. } 1850 \mathrm{C}>\mathrm{T} \\
\text { c. } 4470+10 \_4470+11 \text { ins T }\end{array}$ & $\begin{array}{l}\text { p.(Ala617Val) } \\
{ }^{g} \text { p.(Trp1494Leufs*5) }\end{array}$ & $\begin{array}{l}1.635 \mathrm{e}-5(0) \\
2.031 \mathrm{e}-5(0)\end{array}$ & $\begin{array}{l}\mathrm{T}(0.06) / \mathrm{PD}(0.686) / \mathrm{M} /- \\
-/-/ \mathrm{MF} /-\end{array}$ & $\begin{array}{l}\text { ESEB } \\
\text { ISEB (3), } \\
\operatorname{ESEC~(3)~}\end{array}$ & $\begin{array}{l}\text { rs549230080 } \\
\text { rs754569481 }\end{array}$ \\
\hline
\end{tabular}

CSSA cryptic splicing site activated, DSB donor site broken, ESEB exonic splicing enhancer broken, ESEC exonic splicing enhancer created, ISEB intronic splicing enhancer broken, - not apply

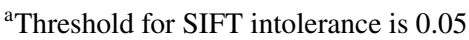

${ }^{\mathrm{b}}$ Polyphen scores: $0.0-0.15$, benign; $0.15-1.0$, possibly damaging; $0.85-1.0$, damaging

${ }^{\mathrm{c}} E V E P$ Ensembl variant effect predictor, $L$ low, $M$ moderate, $M F$ modifier

${ }^{\mathrm{d}}$ Deleterious annotation of genetic variants using neural networks (DANN) score: $0-1,1$ is given to the variants predicted to be the most damaging. $B$ benign, $D$ damaging, $P D$ probably damaging, $T$ tolerated

${ }^{\mathrm{e}} \mathrm{HSF}$ : Human Splicing Finder software (https://www.umd.be/HSF3)

${ }^{\mathrm{f}}$ Frameshift predicted in the open reading frame (ORF) originated by retention of the corresponding intron

${ }^{g}$ Frameshift predicted in the ORF of the new alternative transcript identified in this study, which retains the proximal intron 34

the father was not available for genetic studies, the absence of the CNV in the mother supports the notion that these variants were coinherited with the disease in a recessive fashion (Fig. 1b). The third patient (JG-ASD-33) presented with two compound heterozygous CPAMD 8 nucleotide substitutions (c.2807C $>$ T and c.3991G $>$ T) (Fig. 1a) that were inferred to cause missense variants [p.(Pro936Leu) and p.(Ala1331Ser), respectively]. The former variant did not disrupt any of the A2M family domains, although it affected the amino acid residue Pro936 which shows high evolutionary conservation (Fig. S2), and the latter was positioned in the $\mathrm{A} 2 \mathrm{M}$ thiolester-containing domain (Fig. 2a). The frequency of these variants was very low in gnomAD with no reported homozygous genotypes (Table 1). Prediction of pathogenicity with three programs showed discrepant results. p.(Ala1331Ser) was classified as probably damaging or damaging by two programs, while p.(Pro936Leu) was considered pathogenic by only one (Table 1). In addition, five algorithms contained in the Human Splicing Finder software indicated that variant p.(Ala1331Ser) had the potential to break an exonic splicing enhancer (ESE), resulting in a putative exon skipping with a likely associated severe functional disruption (Table 1). The presence of both variants and their segregation with the disease following a recessive pattern was confirmed by direct Sanger sequencing (Fig. 1b and Fig. S1). Trio WES in the fourth patient (CG-ASD-104) revealed potential pathogenic compound heterozygous variants only in CPAMD 8 (Fig. 1a). The two variants identified in this gene consisted of a novel one-nucleotide deletion (c.2532del) (Table 1) inferred to result in a frameshift close to the $\mathrm{C}$-terminal region of the A2M domain [p.(Lys845Argfs*14), Fig. 2a]. The second alteration was a rare nucleotide substitution (c.2002C $>\mathrm{T} /$ rs200025505) with no reported homozygotes in gnomAD (Table 1) and predicted to result in a PCT (p.(Arg668*)) at the cleavage motif in the bait region (Fig. 2a). The two variants are predicted to be associated with a high functional impact because they would lead to NMD-dependent mRNA degradation, resulting in LoF of the gene and the absence of CPAMD8 protein activity in the patient. The two variants were confirmed by Sanger sequencing (Fig. S1) and segregated with the disease according to a recessive inheritance (Fig. 1b).

In light of the presence of CPAMD 8 variants in CGASD patients we used the same gene panel and filtering algorithm to investigate the possible role of this gene in PCG. To that end, we selected 50 PCG patients from our 
previously reported cohort (Campos-Mollo et al. 2009; López-Garrido et al. 2013). The selected subjects were negative for pathogenic genotypes in CYP1B1, FOXC1, FOXC2 or $M Y O C$. Potential pathogenic CPAMD8 biallelic variants were identified only in patient PCG-30 (Fig. 3a). One allele, p.(Ala617Val), affected the A2M bait region domain (Fig. 2a) and the other one, c.4470+10_4470+11insT, flanked the donor splicing site of intron 34 (Fig. 2b). Both variants had very low frequencies with no reported homozygotes in gnomAD (Table 1). Two out of three bioinformatic tools classified p.(Ala617Val) as a moderate or probably damaging missense variant; in addition, this variant was predicted to break an ESE. Similarly, the intronic nucleotide insertion c.4470+10_4470+11insT also potentially affected

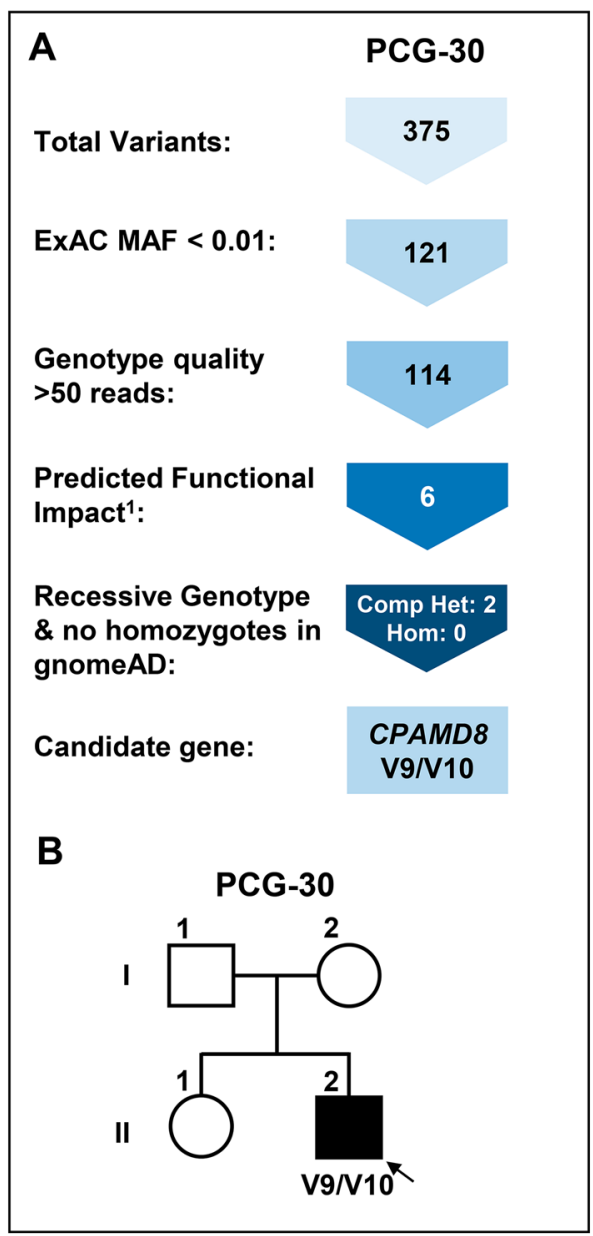

Fig. 3 Variant filtering scheme used for candidate variant identification and pedigree analysis of CPAMD 8 variants in a patient with primary congenital glaucoma. a Gene variants were identified by NGS using a gene panel comprising 121 genes and filtered as indicated in the left to identify candidate variants. b Pedigree of the family. The black symbol indicates the presence of the disease. The arrow in the pedigree shows the index case. + : wild-type allele. ${ }^{1}$ Frameshift, stop gained, start lost, splicing acceptor, and missense variants. V9: p.(Ala617Val), V10: c.4470+10_4470+11insT intronic and/or ESEs (Table 1). The variants were confirmed by Sanger sequencing (Fig. 3b and Fig. S1) but DNA samples from family members were not available for segregation analysis. In addition, we found that seven out of the 50 PCG patients (14\%) carried seven different monoallelic rare CPAMD 8 nucleotide substitutions (Table S1), which were further confirmed by Sanger sequencing (Fig. S4). These variants are described in Suppl. Results.

\section{Clinical features of patients with biallelic CPAMD8 variants}

The four CG-ASD patients who carried biallelic CPAMD8 variants presented variable anterior segment alterations. The mildest phenotype was observed in patient CG-ASG-160, who presented with subtle corectopia and iridocorneal adhesions (Fig. 4) along with very thick corneas and childhoodonset glaucoma (Table 2). This patient had normal zonulas in both eyes with no evidence of ectopia lentis. Case JG-ASD-33 was diagnosed with glaucoma in her teens in Romania, was subjected to trabeculectomy at the age of 31 , developed malignant glaucoma in the RE before receiving treatment at San Carlos Clinical Hospital, and currently suffers from terminal glaucoma in this eye. Eye examination revealed bilateral moderate corectopia with multiple iridocorneal adhesions (Fig. 4). In addition, this subject presented with posterior embryotoxon (Fig. 4) and a bilateral narrow anterior chamber with plateau iris configuration in the left eye (LE) and iatrogenic cataracts (Table 2). Anterior segment malformations present in patient CG-ASD-104 included bilateral corectopia, iris hypoplasia, posterior embryotoxonm, and iridocorneal adhesions (Fig. 4). Iris concavity, iridodonesis, megalocornea, and a deep anterior chamber were additional clinical features of this case (Table 2). This patient had been diagnosed with bilateral congenital glaucoma at the age of 1 , with IOP values before treatment of 20 and $40 \mathrm{mmHg}$ in the $\mathrm{RE}$ and LE, respectively. In addition, she suffered from band keratopathy in the LE secondary to retinal detachment (Fig. 4). Case ASDANI-0066 presented with the most severe phenotype characterized by bilateral iris atrophy, coloboma, megalocornea, increased corneal thickness, bilateral cataracts, and bilateral congenital glaucoma diagnosed at birth (Table 2). The four CG-ASD patients underwent different types of bilateral glaucoma surgery, which included trabeculectomy, goniotomy, and drainage valve implantation (Table 2). Patients JGASD-33 and CG-ASD-ANI-0066 also require ocular drops for adequate IOP control (Table 2). The only PCG patient identified with biallelic CPAMD8 variants was diagnosed 1 week after birth with IOP values of 25 and $30 \mathrm{mmHg}$ in the RE and LE, respectively, and with bilateral optic disc excavation of 0.4 (Table 2). Ocular exploration did not reveal any anterior segment abnormality and there were no signs 


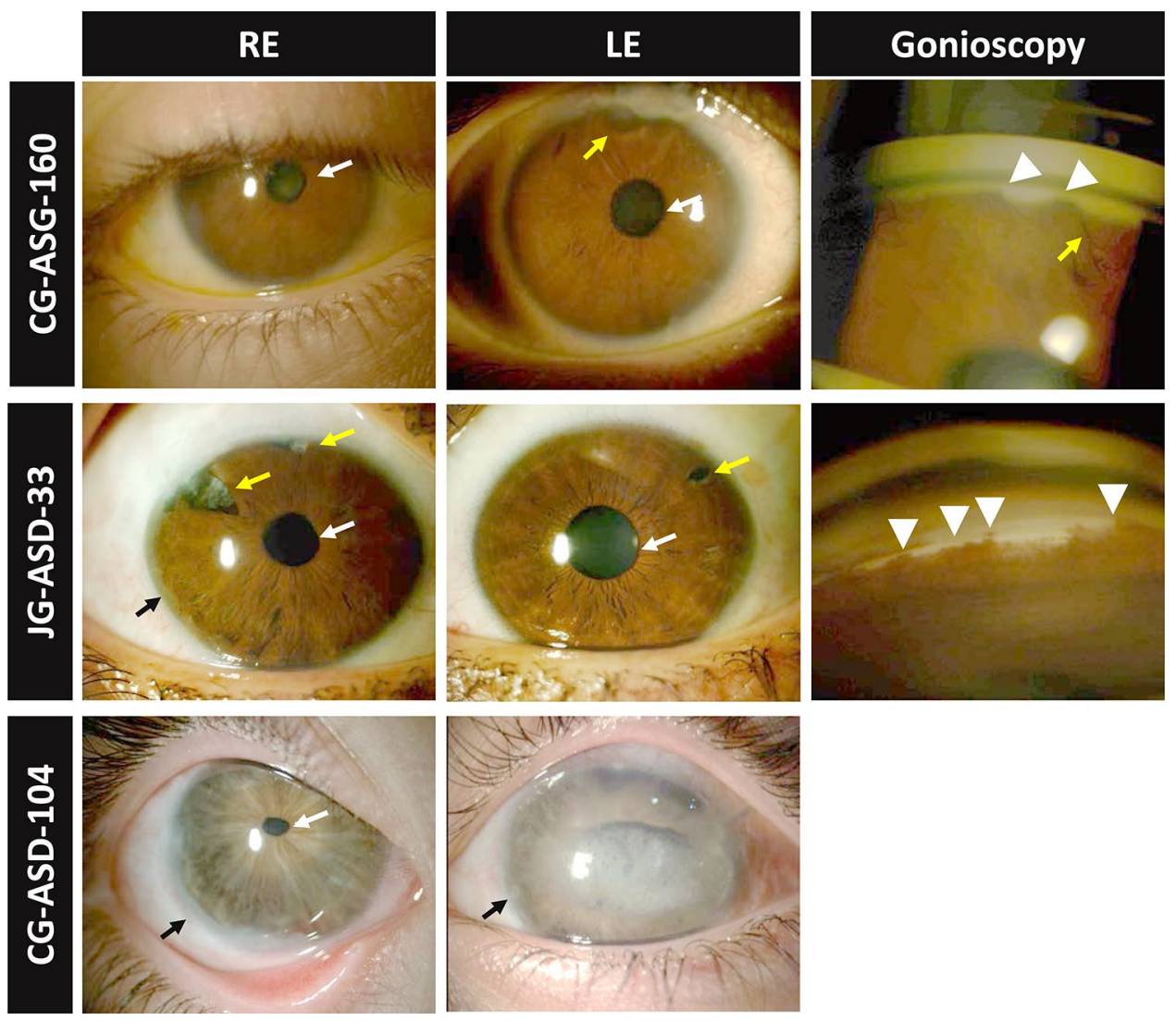

Fig. 4 Ocular phenotypes associated with CPAMD8 variants identified in patients with glaucoma and variable severity of bilateral anterior segment dysgenesis. Phenotype severity increases from patient CG-ASG-160 to CG-ASD-104. Patient CG-ASG-160 shows mild corectopia (white arrows) and iridocorneal adhesions visible under gonioscopy (white arrowheads). Iridectomies are present in the left eye (yellow arrows). Patient JG-ASD-33 presents moderate corectopia (white arrows), iris hypoplasia, and posterior embryotoxon (black

of systemic alterations. This subject required bilateral goniotomy to control IOP. No additional lens or systemic alterations were detected in any of the five patients.

\section{Trabecular meshwork alterations associated with CPAMD8 LoF variants}

A trabeculectomy specimen from the RE of patient CGASD-104 was used for histological analysis. Light microscopy of semithin sections of the anterior chamber angle segment revealed the presence of SC and collector channels (CC) with open lumens and abnormal ciliary muscle (CM) insertion over a quite compact TM (Fig. 5A). Transmission electron microscopy showed the presence of apoptotic cells in the $\mathrm{CM}$, characterized by cell shrinkage and nuclear condensation (Fig. 5B). Endothelial cells of both CC (Fig. 5C) and SC (Fig. 5D) showed vesicles and caveolae, supporting their functionality. The juxtacanalicular tissue (JCT) arrow), along with numerous irodocorneal sinequiae observed gonioscopically (white arrowheads). Iridectomies were present in both eyes (yellow arrows). Patient CG-ASD-104 showed one of the most severe phenotypes, characterized by corectopia (white arrows), evident iris hypoplasia, iridodonesis, and posterior embryotoxon (black arrow). Corneal calcification secondary to retinal detachment was present in the LE. Gonioscopy images were not available, but iridocorneal adhesions were also observed. $L E$ left eye, $R E$ right eye

presented abundant "optically empty spaces", normal proportions of extracellular matrix components (collagen and elastic-like fibers) and apoptotic JCT cells (Fig. 5D). In the $\mathrm{TM}$, most trabecular cells were absent and the few remaining showed clear signs of apoptosis, i.e., condensed chromatin that appears as dark areas in the nucleus of apoptotic cells (Fig. 5E, G, white arrowheads) and apoptotic bodies (Fig. 5E-I, white arrows) which arise during the later phase of apoptosis and consist of cell fragments composed of intact cell membranes containing cytoplasm with tightly packed organelles with or without nuclear fragments (Elmore 2007). Remarkably, the corneoscleral TM (CTM) presented compact trabecular beams with variable thicknesses (Fig. 5E, F) and partially fused at some points (Fig. 5E, F). In addition, the trabecular beams core showed large deposits of coalescent and disorganized fibrillary collagen, abnormal deposits of basement membrane, and irregularly distributed elasticlike fibers (Fig. 5E, F). The uveal TM (UTM) presented 
Table 2 Ophthalmological features of patients with biallelic CPAMD8 variants

\begin{tabular}{|c|c|c|c|c|c|}
\hline Patient & CG-ASD-160 & CG-ASD-ANI-0066 & JG-ASD-33 & CG-ASD-104 & PCG-30 \\
\hline $\begin{array}{l}\text { Age at diagnosis/age } \\
\text { at last ophthalmic } \\
\text { revision }\end{array}$ & Infantile/36 years & Birth/35 years & Juvenile/25 years & Birth/17 years & $1 \mathrm{w} / 8$ years \\
\hline Inheritance & $\mathrm{AR}$ & AR & $\mathrm{AR}$ & AR & $\mathrm{AR}$ (putative) \\
\hline BCVA (RE/LE) & $0.25 / 1$ & $0.5 / 0.5$ & $0.66 / 1$ & $0.2 / \mathrm{LP}$ & $0.7 / 0.5$ \\
\hline Refraction (RE/LE) & $-12.00 /-9.00$ & $-4.25 /-4.0$ & $-3.50 /+1.50$ & $-6.00 /-18.00^{\mathrm{a}}$ & NA \\
\hline $\begin{array}{l}\text { Axial length (mm) (RE/ } \\
\text { LE) }\end{array}$ & $29.38 / 26.69$ & ND & $22.67 / 22.23$ & $26.16 / 36.79$ & NA \\
\hline $\mathrm{AC}$ depth (mm) (RE/LE) & $4.16 / 4.59$ & NA & Narrow/narrow & $5.03 / 3.26$ & NA \\
\hline $\begin{array}{l}\text { Lens thickness (RE/LE) } \\
\quad(\mathrm{mm})\end{array}$ & 5.13/4.87 (Clear lens) & NA (B) (CS) & $\begin{array}{l}\text { 5.13/4.87, iatrogenic } \\
\text { cataract }(\mathrm{B})\end{array}$ & Clear lens/Aphakic & NA \\
\hline Iris & Mild C & Coloboma and AS (B) & $\begin{array}{l}\text { Moderate C and AS (B), } \\
\text { PI (B) }\end{array}$ & $\begin{array}{l}\text { AS, C, ID, IH and IC } \\
\text { (B) }\end{array}$ & Normal \\
\hline $\begin{array}{l}\text { Central corneal thickness } \\
(\mu \mathrm{m})(\mathrm{RE} / \mathrm{LE})\end{array}$ & $665 / 640$ & $600 / 600$ & $539 / 559$ & NA & NA \\
\hline Corneal morphology & Normal & $\mathrm{MC}(\mathrm{B})$ & $\mathrm{PE}(\mathrm{B})$ & $\mathrm{MC}$ and $\mathrm{PE}(\mathrm{B})$ & Normal \\
\hline Posterior segment & Coroidosis, miopía & Normal & & & Normal \\
\hline Glaucoma & $+(\mathrm{B})$ & $+(\mathrm{B})$ & $+(\mathrm{B})$ & $+(\mathrm{B})$ & $+(\mathrm{B})$ \\
\hline $\begin{array}{l}\text { IOP at diagnosis (mm } \\
\mathrm{Hg})(\mathrm{RE} / \mathrm{LE})\end{array}$ & NA & NA & NA & $20 / 40$ & $25 / 30$ \\
\hline $\begin{array}{l}\text { Last IOP (mm Hg) (RE/ } \\
\text { LE) }\end{array}$ & $20 / 18$ & $18 / 18$ & $20 / 20$ & $16 / 16$ & NA \\
\hline $\begin{array}{l}\text { C/D ratio at diagnosis } \\
\text { (RE/LE) }\end{array}$ & $0.8 / 0.8$ & NA & NA & $0.6 / 0.6$ & $0.4 / 0.4$ \\
\hline $\begin{array}{l}\text { Last cup/disc ratio (RE/ } \\
\text { LE) }\end{array}$ & $0.8 / 0.8$ & NA & $0.9-0.3$ & $0.6 / 0.9$ & NA \\
\hline $\begin{array}{l}\text { Glaucoma surgery (num- } \\
\text { ber, laterality) }\end{array}$ & $\mathrm{G}(1, \mathrm{~B}), \mathrm{T}(1 \mathrm{RE} / 2 \mathrm{LE})$ & $\mathrm{G}(1, \mathrm{~B}), \mathrm{Cs}(1, \mathrm{~B})$ & $\mathrm{T}(1, \mathrm{~B})$ & $\begin{array}{l}\mathrm{G}(3, \mathrm{~B}) ; \mathrm{T}(1, \mathrm{~B}) ; \mathrm{AV} \\
\quad(1, \mathrm{LE}) ; \mathrm{V}(\mathrm{B})\end{array}$ & $\mathrm{G}(1, \mathrm{~B})$ \\
\hline $\begin{array}{l}\text { Number of antiglaucoma } \\
\text { drugs (RE/LE) }\end{array}$ & $1 / 1$ & $2 / 2$ & $0 / 4$ & $0 / 0$ & NA \\
\hline
\end{tabular}

$A C$ anterior chamber, $A V$ Ahmed valve, $A S$ anterior synequia, $B$ bilateral, $B C V A$ best corrected visual acuity, $C$ corectopia, $C / D$ cup/disc, $C S$ cataract surgery, $G$ goniotomy, $I D$ iridodonesis, $I H$ iris hypoplasia, $I C$ iris concavity, $I O P$ intraocular pressure, $L P$ light perception, $M C$ megalocornea $N A$ no available, $N E$ no evaluable, $P E$ posterior embryotoxon, $P I$ plateau iris, $R E$ right eye, $L E$ left eye, $T$ trabeculectomy, $V$ vitrectomy, $y$ year, $w$ week

${ }^{a}$ Before vitrectomy

intertrabecular spaces (Fig. 5G) with partially fused trabecular beams (Fig. 5G). Trabecular beams core showed the accumulation of elastic-like fibers and densely packed and disorganized fibrillary collagen (Fig. 5G-I) arranged in onion-like layers (Fig. 5G), scarce non-fibrillar type VI collagen and thick basement membrane (Fig. 5H, I).

\section{Functional evaluation of the identified variants}

Fresh blood samples from family CG-ASD-104 were used to investigate the effect of the two identified variants on CPAMD 8 mRNA levels by qRT-PCR. CPAMD 8 transcript levels were reduced to approximately $30 \%$ of those observed in control subjects who did not carry any pathogenic variant in this gene (Fig. 6), demonstrating the functional disruption of CPAMD8 in this patient. Unexpectedly, the mRNA levels in heterozygous carriers of the pathogenic variants were similar to those of controls, indicating the existence of the transcriptional adaptation or compensation of this gene to pathogenic mutations triggered by mRNA degradation (El-Brolosy et al. 2019).

Fresh blood samples were not available from families CG-ASD-160 and PCG-30. Therefore, we evaluated in vitro the splicing effect of variants predicted to affect intron processing using an assay based on a modified version of the $\alpha$-globin-fibronectin EDB minigene (Cheong et al. 2016; Pagani et al. 2003). To that end, genomic DNA fragments containing these variants and their corresponding wild-type alleles were cloned from the genomic DNA of patients (Fig. 7A and Fig. S5A) and expressed in HEK-293T cells as described in the Methods section. PCR analysis of minigene transcripts derived from patient CG-ASD-160 

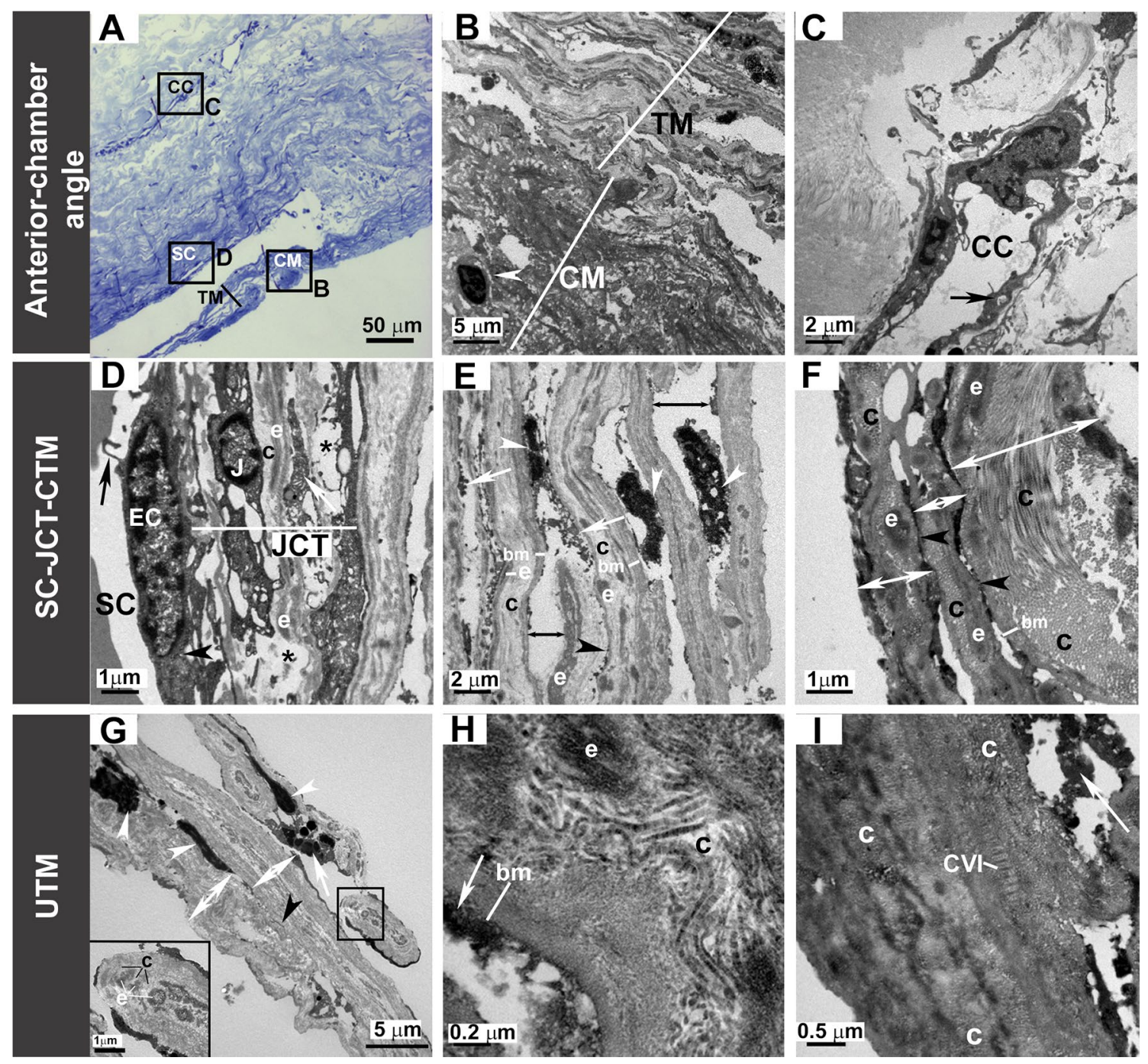

Fig. 5 Anterior chamber angle dysgenesis in CPAMD8-associated CG. Trabeculectomy from the right eye of patient CG-ASD-104. A Light microscopy of the anterior chamber angle segment. Abnormal insertion of the CM; compact TM; open lumens of SC and CC. Squares: areas magnified in panels $\mathbf{B}, \mathbf{C}$, and $\mathbf{D}$. B-I Transmission electron microscopy. B Aberrant CM position over the TM. Apoptotic $\mathrm{CM}$ cell (white arrowhead). C CC with open lumen and vacuoles in endothelial cells (black arrow). D Vacuoles (black arrow) and vesicles (black arrowhead) in an endothelial cell of the SC. JCT cells (J), elastic-like fibers (e), fibrillary collagen (c), optically empty spaces (*) and apoptotic bodies (white arrow) in the JCT. E CTM with apoptotic trabecular cells (white arrowheads) and apoptotic bodies (white arrow). Abundant fibrillary collagen (c) and elastic-like fibers (e). Intertrabecular spaces (black double arrow) alternate with fused trabecular beams (black arrowhead). F Trabecular beams filled with densely packed and disorganized fibrillary collagen (c), elastic-like fibers (e) and thick basement membrane (bm). Trabecular beams (white double arrow) present variable thickness and partial fusions (black arrowhead). G UTM with intertrabecular spaces (white double arrow), fused trabecular beams (black arrowhead), apoptotic trabecular cells (white arrowhead) and apoptotic bodies (white arrow). In cross-sectioned trabecular beams (inset in G) elastic material (e) and fibrillary collagen (c) are arranged in onion-like layers. H, I UTM trabecular beam with apoptotic cell debris (white arrow), abundant densely packed and disorganized fibrillary collagen (c), thickened basement membrane (bm), scarce collagen VI (CVI), and elastic-like fibers (e). $C C$ collector channel, $C M$ ciliary muscle, $C T M$ corneoscleral trabecular meshwork. EC endothelial cell, JCT juxtacanalicular tissue, $S C$ Schlemm's canal, TM trabecular meshwork, UTM uveal trabecular meshwork. Scale bars: $50 \mu \mathrm{m}$ in $\mathbf{A} ; 5 \mu \mathrm{m}$ in $\mathbf{B}$ and G; $2 \mu \mathrm{m}$ in $\mathbf{C}$ and $\mathbf{E} ; 1 \mu \mathrm{m}$ in $\mathbf{D}, \mathbf{F}$ and inset in $\mathbf{G} ; 0.2 \mu \mathrm{m}$ in $\mathbf{H}$ and $0.5 \mu \mathrm{m}$ in $\mathbf{I}$ (c.1758+1_1758+4del) using EDB specific primers p3/ p4 showed a 482 bp band (Fig. 7B, band 1). By contrast, the wild-type minigene version produced a 428-bp amplicon (Fig. 7B, band 2). Direct Sanger sequencing revealed that bands 1 and 2 corresponded to an aberrant splicing product that retained 54 nucleotides from intron 15 and to the normally spliced exon 15, respectively (Fig. 7B and Fig. S6A). This result indicates that elimination of the canonical splicing donor signal by variant c.1758+1_1758+4del leads to the activation of an intron 15 cryptic splicing donor 


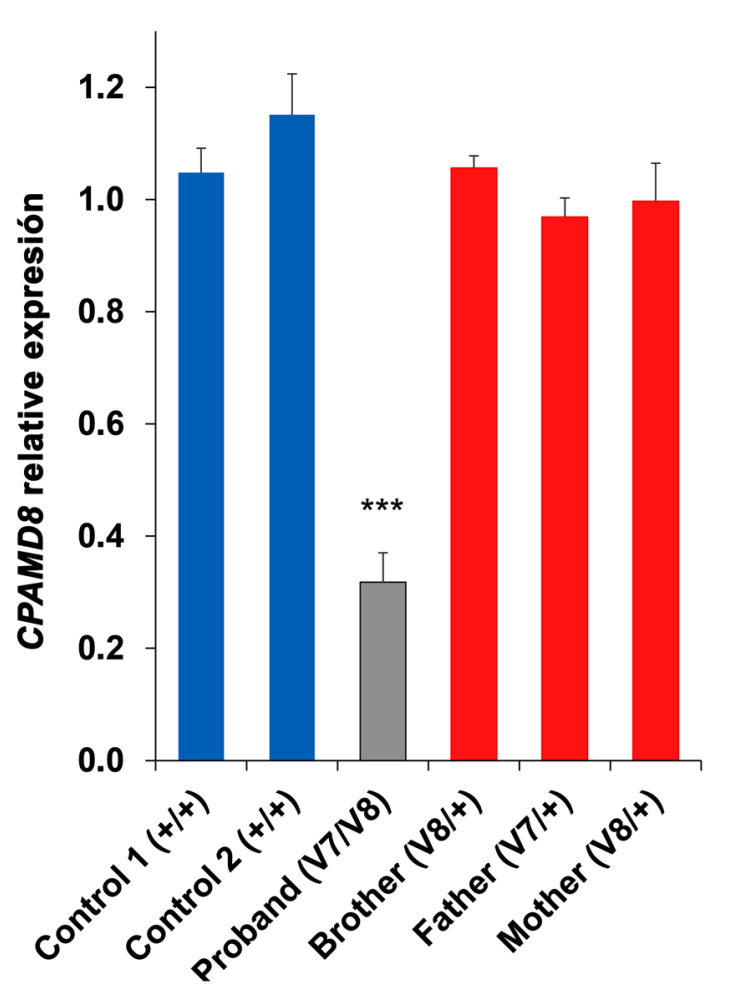

Fig. 6 Reduced CPAMD8 mRNA levels in the CG-ASD-104 patient. The mRNA levels were measured by qRT-PCR using RNA purified from peripheral blood leukocytes as described in the "Methods" section. The results are expressed as a relative expression to control 1 (normal subject with no pathogenic CPAMD8 variants). Controls 1 and 2 are unrelated healthy subjects. + : wild-type allele. V7: p.(Lys845fs*13). V8: p.(Arg668*). Asterisks indicate statistical significance compared to the control: $p<0.001$ (***). Statistical significance was calculated by ANOVA

site, resulting in the partial retention of intron 15 (Fig. 7B). Bioinformatic analysis confirmed the presence of a potential splicing donor signal at position +58 of the wild-type intron (Fig. 7C). Therefore, partial retention of intron 15 creates a new open reading frame with a PCT in the fifth codon (Fig. 7C, asterisk) that may finally lead to NMDdependent mRNA degradation. Other minor amplicons were also observed in the mutant minigene assay, indicating the existence of other secondary uncharacterized transcripts (Fig. 7B). Approximately equal-intensity amplicons were obtained from control- and patient-derived minigenes, using vector-specific primers $\mathrm{p} 1 / \mathrm{p} 2$, indicating no significant expression differences between the two DNA constructs (Fig. 7B, band 3). Minigene analysis of the intronic variant identified in patient PCG-30 (c.4470+10_4470+11 insT) did not show any mRNA splicing effect under the experimental conditions used but did reveal a previously unidentified alternative transcript, which partially retained intron 34 and, thus, might be affected by the identified nucleotide insertion (Suppl. Results, Figs. SF5A, B, S6A). The existence of this alternative transcript was demonstrated in peripheral human leukocytes of a control subject (Suppl. Results, Fig. S5C-E and Fig. S6C). Interestingly, these results show that the nucleotide insertion (c.4470+10_4470+11insT) becomes a coding variant (c.4480_4481InsT) in the alternative mRNA, resulting in frameshift and the premature translation termination of the new open reading frame (p.(Trp1494Leufs*5), Fig. S5E, blue asterisk). Thus, this variant will underlie specific NMD-dependent degradation of the new alternative transcript, supporting its pathogenicity. Further characterization of the alternative mRNA is required to completely understand the effect of this nucleotide insertion.

No functional assays were available to evaluate the effect of the three missense variants identified in the study.

\section{Presence of CPAMD8 in adult human ocular tissues}

To the best of our knowledge the presence of CPAMD8 protein in adult human ocular tissues has not been studied. Western blot analysis of ocular fluids with three different commercial antibodies showed the presence of $\mathrm{N}$ - and C-terminal CPAMD8 fragments in aqueous humor (AH) and vitreous humor (Suppl. Results and Fig. S7). Next, we used one of these antibodies (Ab1) to study the distribution of this protein by confocal FIHC in adult human ocular anterior segment tissues. CPAMD8 was detected in different epithelial and muscular tissues. In fact, we observed intense cytoplasmatic granular signals in the non-pigmented ciliary epithelium (NPCE) (Fig. 8A). Iris muscles (sphincter and dilator) (Fig. 8B) and especially longitudinal fibers of the CM (Fig. 8C) were also positively labeled. In addition, CPAMD8 was detected in the subcapsular lens epithelium, lens fibers (Fig. 8D), corneal epithelium, keratocytes, and corneal endothelium (Fig. 8E-G, respectively). The specificity of these signals was supported by their absence in the corresponding negative controls (Fig. S8). Interestingly, the protein was not detected in the trabecular meshwork or SC (Fig. S9).

\section{Expression of cpamd8 in zebrafish ocular tissues}

To analyze the function of CPAMD8 in vivo and to evaluate its role in congenital glaucoma and ASD we used zebrafish as an animal model. DNA sequence homology analysis revealed a unique CPAMD8 zebrafish orthologue located on chromosome 22, with well-conserved intron-exon and protein domain organization (Fig. S10A and B, respectively). The two orthologue proteins also show a high amino acid sequence identity (65.5\%). Ocular cpamd8 expression was analyzed in zebrafish embryos by FWIHC using Ab1 at $96 \mathrm{~h}$ post fertilization (hpf). The zebrafish epitope recognized by this antibody is $62 \%$ amino acid-sequence-identical to the corresponding human epitope. Three-dimensional reconstruction by confocal laser scanning microscopy revealed 


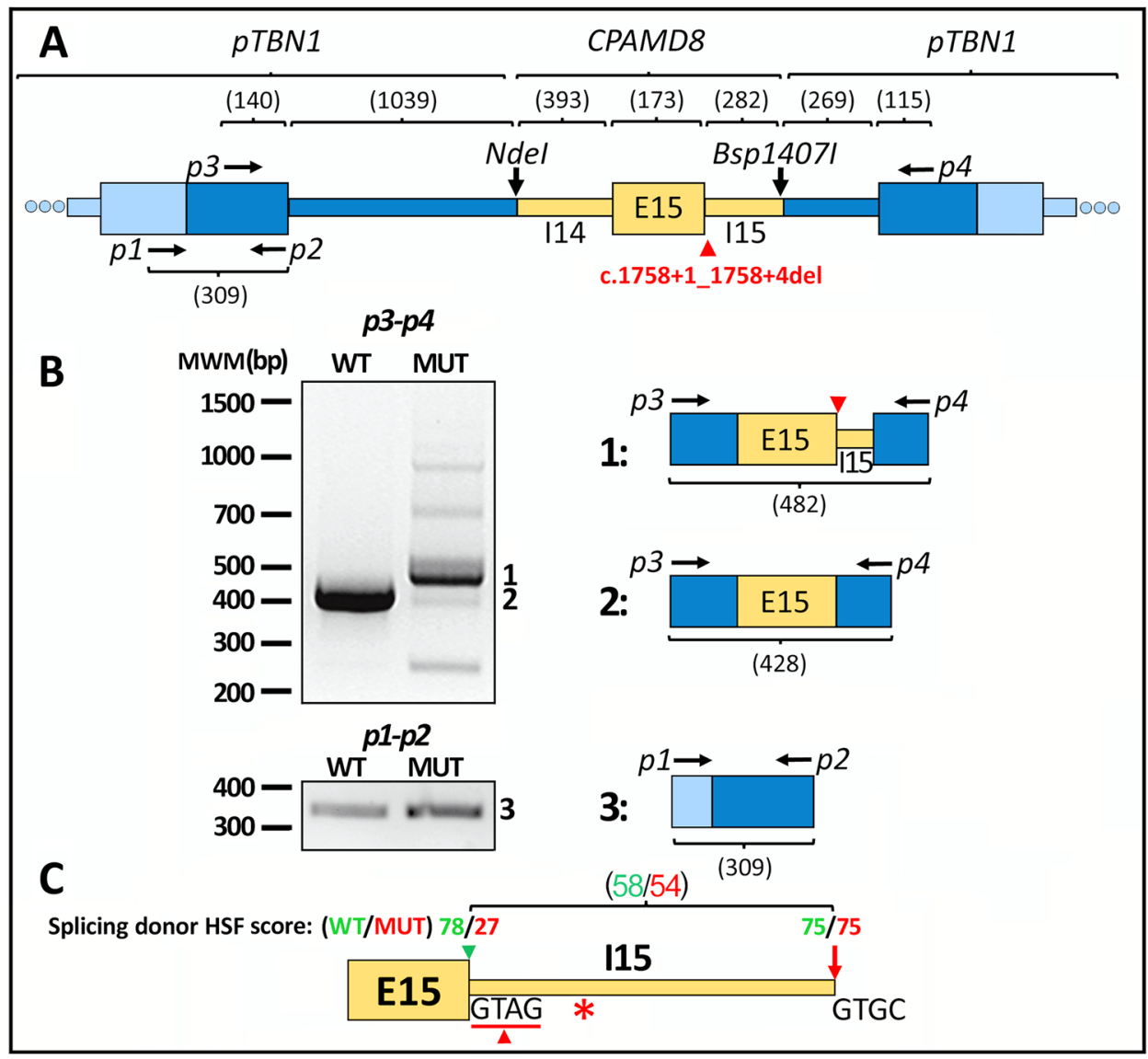

Fig. 7 Functional analysis of the c.1758+1_1758+4del CPAMD8 variant identified in patient CG-ASD-160. A Scheme of the cDNA construct used for in vitro mRNA splicing assay using the pTBN1 vector (blue region). Wild-type and mutant (c.1758+1_1758+4del) CPAMD 8 genomic fragments are indicated in yellow. Vertical black arrows: restriction endonuclease sites used for cloning. B Agarose electrophoresis analysis of wild-type (WT) and mutant (MUT) transcripts generated by RT-PCR using primers p3-p4 (left panel). Transfection efficiency was assessed by PCR amplification using minigene vector primers p1-p2 (band 3). The different amplicons were analysed by Sanger sequencing (Fig. S6A) and the inferred organiza-

specific periocular labeling, localized in the optic cup edge with superficial positive mesenchymal-like cells in the dorsoposterior quadrant of the eye and irregularly distributed in the periphery of the ocular globe (Fig. 9A). Observation of two representative confocal optical sections clearly showed cpamd8-positive cells irregularly located on the external edge of the optic cup and surrounding the ocular globe (Fig. 9B, C). The absence of green signals in the negative control supports the labeling specificity (Fig. S11). These results were confirmed by FIHC on frozen tissue sections, which also showed cpamd8 positive signals in the developing dorsal and ventral iridocorneal angles, in the iris, between the lens and the optic cup surface, and periocularly distributed around the ocular globe (Fig. S12, top panels), in positions that may correspond to periocular mesenchyme. tion is shown on the right. C Prediction of a potential splicing donor signal (GTGC) by the Human Splicing Finder (HSF) software. HSF scores for wild-type and mutant alleles are indicated in green and red, respectively. Note that HSF values for the canonical and alternative splicing sites are similar ( 78 and 75 , respectively). Strong sites present values higher than 80 . Green arrowhead: canonical splicing cleavage site. Red arrowhead: position of the c.1758+1_1758+4del variant. Red asterisk: position of a PCT in the fifth codon of the mutant reading frame. Nucleotides of the canonical splicing signal (GTAG), which are deleted in the mutant allele, are indicated

Again, the absence of green labeling in the negative controls indicated the specificity of the detected signals (Fig. S12, bottom panels).

\section{F0 zebrafish phenotypes resulting from CRISPR/ Cas9 cpamd8 inactivation}

To evaluate whether cpamd8 plays a role in early ocular development in zebrafish, we inactivated this gene using CRISPR/Cas9 genome editing. We generated two independent $100 \mathrm{bp}$-deletions with crRNA pairs targeting exons 4 (cpamd $8^{\mathrm{ex} 4}$ ) and 25 (cpamd8 $8^{\mathrm{ex} 25}$ ) (Fig. S10A, crRNAE4a/ crRNAE4b, and crRNAE25a/crRNAE25b) as described in the "Methods" section. This approach has been demonstrated to be a rapid and efficient strategy for in vivo evaluation 

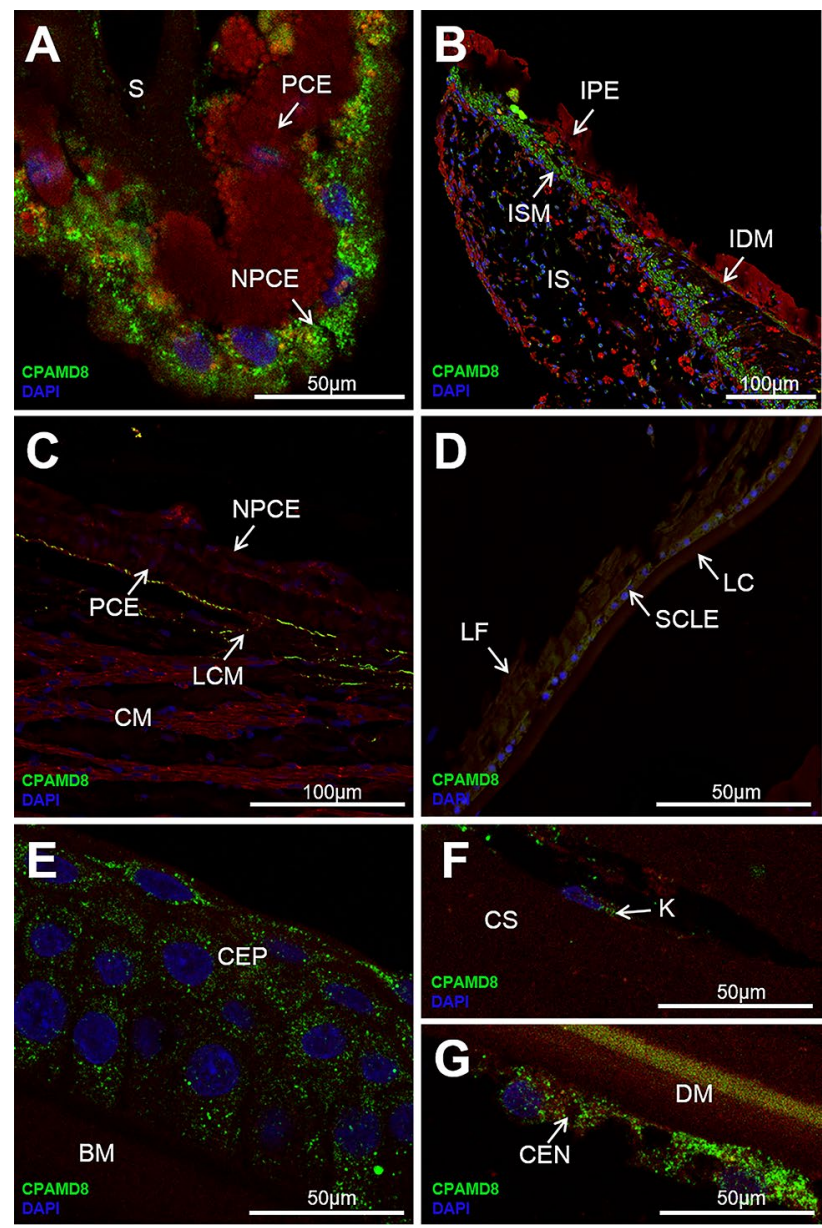

Fig. 8 Localization of CPAMD8 protein in adult human ocular anterior segment tissues. For fluorescent immunohistochemistry, histological sections $(3 \mu \mathrm{m})$ were incubated with either a rabbit anti-human CPAMD8 primary antibody (HPA031328; Sigma) and a Cy2-conjugate donkey anti-rabbit secondary antibody (green signal) (1:200) (A-G) or with only a secondary antibody as a negative control (1:1000) (Fig. S8). Confocal wide-field micrographs of ciliary processes $(\mathbf{A})$, iris $(\mathbf{B})$, pars plana/ciliary muscle $(\mathbf{C})$, lens epithelium (D), detailed images of the corneal epithelium $(\mathbf{E})$, corneal stroma and keratocytes $(\mathbf{F})$, and corneal endothelium $(\mathbf{G})$. Scale bars correspond to $50 \mu \mathrm{m}$ in panels $\mathbf{A}, \mathbf{D}, \mathbf{E}, \mathbf{F}, \mathbf{G}$, and to $100 \mu \mathrm{m}$ in panels $\mathbf{B}$ and $\mathbf{C}$. Red signals correspond to tissue autofluorescence. $B M$ Bowman's membrane, $C E N$ corneal endothelium, $C E P$ corneal epithelium, $C M$ ciliary muscle, $C S$ corneal stroma, $D M$ Descemet's membrane, $I D M$ iris dilator muscle, IPE iris pigment epithelium, $I S$ iris stroma, $I S M$ iris sphincter muscle, $K$ keratocyte, $L C$ lens capsule, $L C M$ longitudinal fibers of the ciliary muscle, $L F$ lens fibers, $N P C E$ non-pigmented epithelium, $P C E$ pigmented epithelium, $S$ stroma, $S C L E$ subcapsular lens epithelium

of LoF-associated phenotypes (Bhattacharya et al. 2015). The phenotypic evaluation was carried out using 50-200 F0 mosaic embryos in each experiment. At 96 hpf, approximately $1 \%$ of non-injected embryos died and only $3 \%$ of tracrRNA-injected embryos (negative control) showed lethal phenotypes characterized by generalized edema and aberrant morphology and they were classified as non-specific phenotypes (Fig. 10A). Lethal phenotypes increased to $15 \%$ and $6 \%$ in cpamd $8^{\text {ex4}}$ - and cpamd $8^{\text {ex25}}$-targeted embryos, respectively. We observed a parallel decreased in normal phenotypes from $97 \%$ in control embryos to $26 \%$ and $60 \%$ in cpamd $8^{\text {ex4}}$ - and cpamd $8^{\text {ex } 25}$-targeted embryos, respectively (Fig. 10A). In addition, three similar abnormal phenotypes (Ph-1, Ph-2 and Ph-3, Fig. 10A) were present in cpamd $8^{\text {ex4 }}$ - and cpamd $8^{\text {ex } 25}$-targeted embryos, which were classified according to the increasing degree of microphthalmia, pharyngeal maldevelopment, and pericardial, periocular and brain edema (Fig. 10B, C). The proportion of these phenotypes ranged from 14 to $25 \%$ in cpamd $88^{\text {ex }}$-targeted embryos and from 7 to $25 \%$ in cpamd 8 ex 25 -targeted embryos (Fig. 10A). Most of these embryos died at $7 \mathrm{dpf}$. These results indicate that the observed developmental alterations are not caused by off-target DNA cleavage. Moreover, these phenotypes were not observed in either tracrRNA-injected or uninjected embryos, indicating that they were specific. Immunohistochemical analysis of the three phenotypes revealed the progressive reduction of cpamd8 protein in periocular tissues, which correlated with the severity of ocular alterations, thereby supporting both correct cpamd8 inactivation and the specificity of the anti-CPAMD8 antibody (Fig. S13). Cpamd8 mosaic disruption in the CRISPR/Cas9 injected embryos was confirmed by PCR analysis (Suppl. Results and Fig. S14). Next, we examined the structure of ocular tissues involved in glaucoma associated with phenotypes $\mathrm{Ph}-1$ to $\mathrm{Ph}-3$. Toluidine-blue-stained head sections of 96-hpf embryos confirmed progressive microphthalmia and showed increased spaces surrounding the brain and eyes filled with amorphous material, particularly in $\mathrm{Ph}-1$ and $\mathrm{Ph}-2$ embryos, which indicates the existence of edema (Fig. $11 \mathrm{~K}, \mathrm{M}$ ). $\mathrm{Ph}-2$ and $\mathrm{Ph}-3$ embryos were also characterized by pharyngeal cartilage underdevelopment (Fig. 11M, Q). Moreover, detailed observation of the anterior segment showed progressive iridocorneal dorsal and ventral angle hypoplasia (Fig. 11), with a decreased number of iris stroma cells, i.e., iridophores, xantophores and mesenchymal cells (Fig. 11, "Dorsal Angle" and "Ventral Angle" panels). Corneal epithelium cells presented increased size with apparent cell swelling from Ph-1 to Ph-3 (Fig. 111, P, T). Transmission electron microscopy of dorsal and ventral iridocorneal angles demonstrated the presence of the primordial anterior chamber space in wild-type and wild type-like embryos (Fig. 12B, C, G-H, respectively). Phenotypes Ph-1 to Ph-3 presented a reduction in this primordial space (in both the dorsal and ventral regions), which, in addition, was increasingly filled with cellular material, correlating with phenotype severity (Fig. 12L, M, Q, R, V and W). Irregular iridophore-reflecting platelets correlated with the severity of the phenotype and were especially evident in phenotypes Ph-2 and Ph-3 (Fig. 12Q, V, R, and W). The two-cell-layer corneal epithelium was gradually thickened from phenotype 


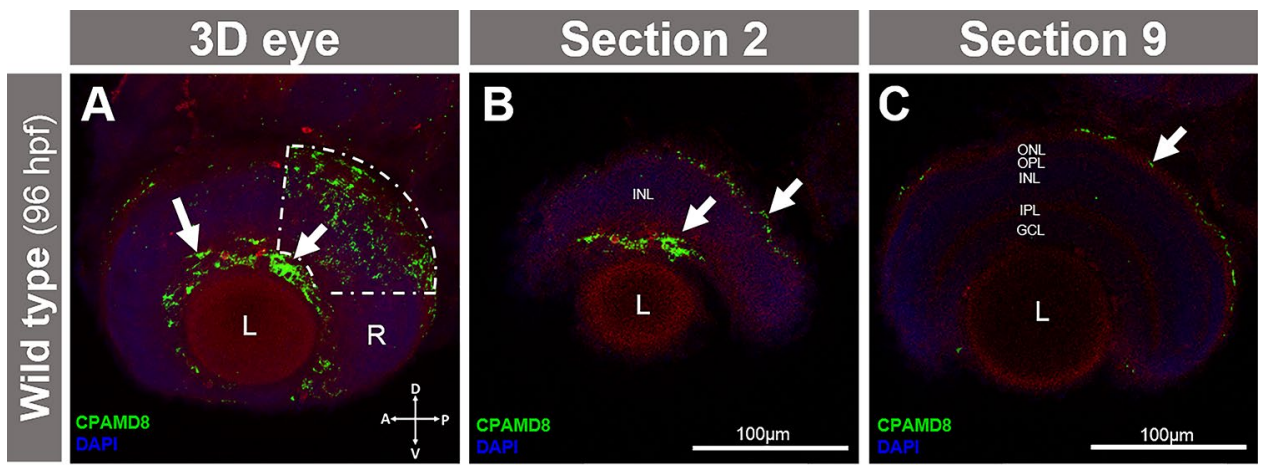

Fig. 9 Fluorescent whole-mount immunohistochemical detection of cpamd8 in the embryonic zebrafish eye (96hpf). The embryos were incubated with either rabbit anti-human CPAMD8 primary antibody (HPA031328; Sigma) (1:200) and Cy2-conjugated donkey anti-rabbit secondary antibody $(1: 1500)$ (A-C) or only a secondary antibody as a negative control (D-F). A Three-dimensional reconstruction from z-stack scanned confocal microscopy images $(144 \mu \mathrm{m})$ of the eye. B, C Optical sections 2 and 9, from the exterior ocular surface, were selected from z-stack images to show the precise localization of the green signal between the optic cup surface and lens (arrow) and in the periocular mesenchyme surrounding the ocular globe (arrowhead). Red: tissue autofluorescence. Discontinuous line: Cpamd8 positive signals in the dorsoposterior quadrant of the eye. The cross indicates the position of the embryonic axes ( $D$ dorsal, $P$ posterior, $V$ ventral, $A$ anterior). The image is representative of the result observed in 10 embryos. $I N L$ inner nuclear layer, $I P L$ inner plexiform layer, $L$ lens, $O N L$ outer nuclear layer, $O P L$ outer plexiform layer, $R$ retina

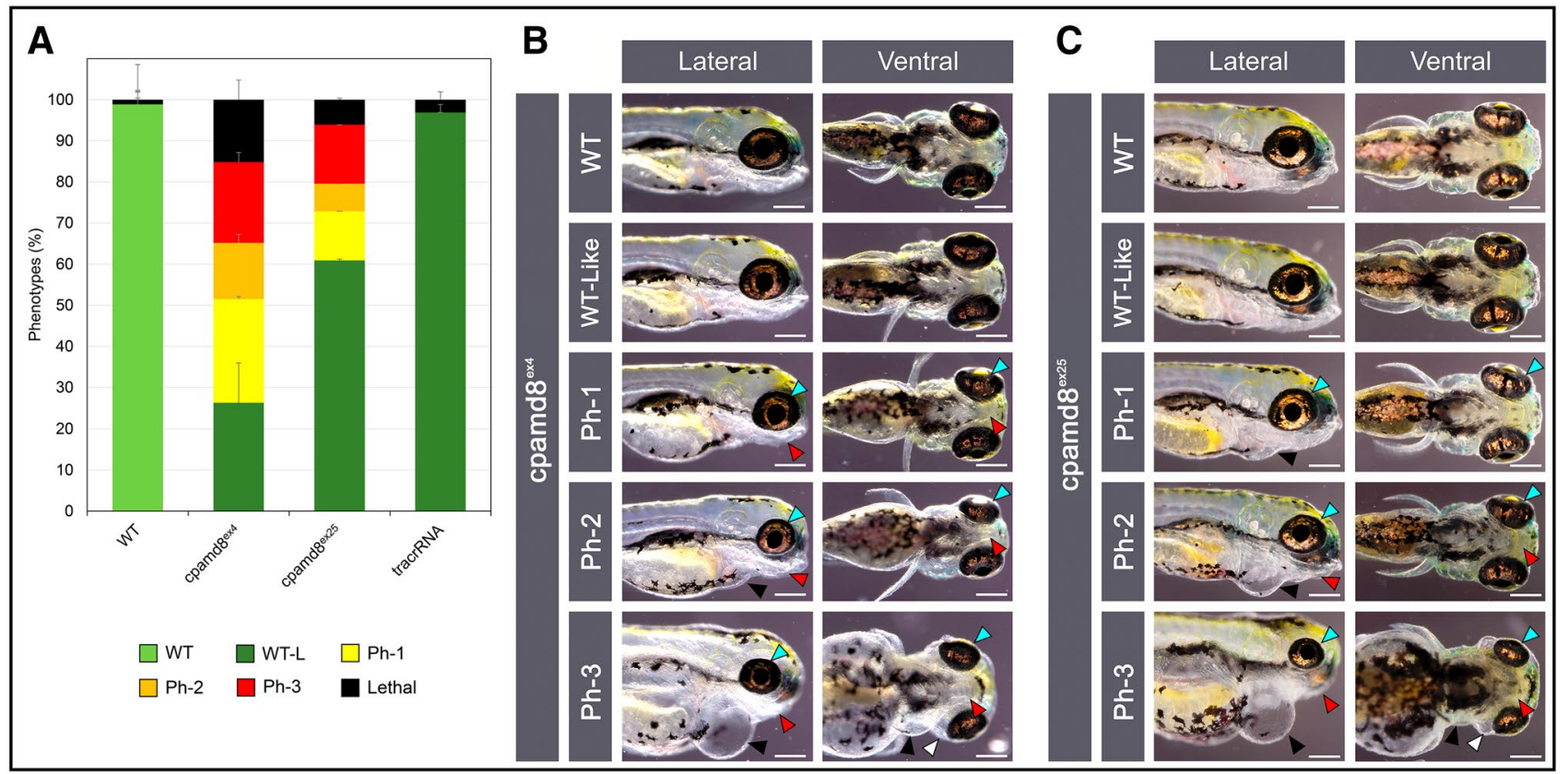

Fig. 10 Analysis of F0 zebrafish phenotypes resulting from CRISPR/ Cas9 cpamd8 inactivation (96-hpf). Zebrafish embryos were microinjected with CRISPR/Cas9 ribonucleoprotein complexes targeting cpamd 8 exon 4 (cpamd $\left.8^{\text {ex } 4}\right)$ or exon 25 (cpamd $8^{\text {ex } 25}$ ). Embryos microinjected with Cas9/tracrRNA ribonucleoprotein complexes and no crRNA were used as controls (tracrRNA). Untreated wildtype embryos were also used as an additional control (WT). Treated embryos (F0) were classified into four phenotypes (WT-like, Ph-1, Ph-2, and Ph-3) according to increasing severity. A Proportion of
F0 zebrafish phenotypes. Values correspond to the mean \pm SEM of triplicate experiments with 50 to 250 embryos per experiment. Phenotypes resulting from targeting of cpamd8 exon 4 (B) or exon 25 (C). Lateral and ventral brightfield micrographs show similar abnormal phenotypes resulting from the targeting of the two exons, including microphthalmia (blue arrowheads), jaw maldevelopment (red arrowheads), and pericardial and periocular edemas (black and white arrowheads, respectively). Scale bars represent $200 \mu \mathrm{m}$ 

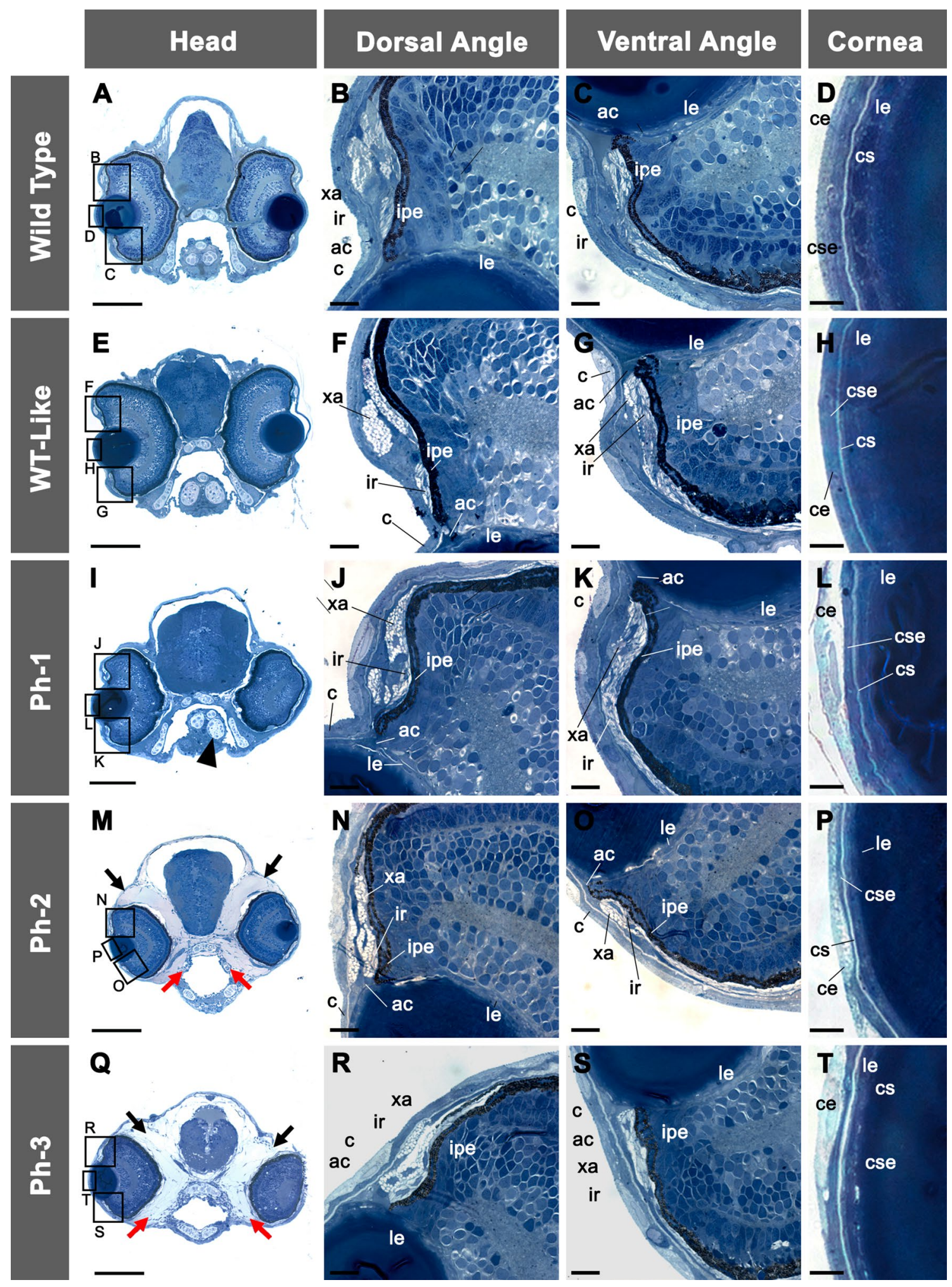

Ph-1 to Ph-3 (Fig. 12N, S, X). The most superficial corneal epithelial cell layer appeared edematous in phenotypes $\mathrm{Ph}-1$ to $\mathrm{Ph} 3$, with swelling of intracellular organelles (Fig. 12N, $\mathrm{S}, \mathrm{X}$, arrowhead), suggestive of apoptosis. The subepithelial cell layer showed an irregular contour (Fig. 12N, S, X, cse). Thickening of the corneal stroma was evident, particularly in Ph-3 embryos (Fig. 12X, Y, cs). Collagen fibers in the corneal stroma of wild-type embryos were regularly arranged in regular 7-9 layers (Fig. 12E, J). By contrast, we observed abnormal ECM characterized by irregular collagen 
४Fig. 11 Histological analysis of F0 zebrafish phenotypes resulting from CRISPR/Cas9 cpamd8 inactivation (96-hpf). A, E, I, M, Q Semi-thin $(500 \mathrm{~nm})$ transverse head sections were stained with Toluidine blue. WT wild type, WT-L wild type-like, $P h-1$ phenotype $1, P h 2$ phenotype $2, P h 3$ phenotype 3 . Progressive microphthalmia and enlarged intracranial and periocular spaces filled with amorphous material (black arrows), correlate with the severity of phenotypes. Underdevelopment of pharyngeal cartilages, ceratohyal (arrowhead), and the palatoquadrate (red arrows) are also present in phenotypes Ph-2 and Ph-3. Scale bar: $100 \mu \mathrm{m}$. The squares and rectangles indicate the areas of the images magnified in the indicated panels. B, C The development of wild-type iridocorneal dorsal and ventral angles shows normal pigment cell stratification in the iris with xantophores and idridophores in the stroma. D The cornea epithelium, subepithelium, stroma, and endothelium in contact with the anterior lens epithelium are observed in wild-type embryos. Progressive anterior angle chamber reduction and hypoplasia of dorsal $(\mathbf{F}, \mathbf{J}$, $\mathbf{N}, \mathbf{R})$ and ventral $(\mathbf{G}, \mathbf{K}, \mathbf{O}, \mathbf{S})$ iris stroma, affecting iridophores and xantophores, correlates with increasing phenotype severity (Ph-1 to $\mathrm{Ph}-3)$. (H, L, P, T). Corneal epithelial swelling and stroma thickening increase with phenotype severity ( $\mathrm{Ph}-1$ to $\mathrm{Ph}-3)$. Ir iridophores, $x a$ xantophores, ipe iris pigmented epithelium, $a c$ anterior chamber, $c$ cornea, ce corneal epithelium, cse corneal subepithelium, $c s$ corneal stroma, $c n$ corneal endothelium, le lens. Scale bar in "Eye" panels: $50 \mu \mathrm{m}$. Scale bar in "Dorsal Angle" and "Ventral Angle" panels: $4 \mu \mathrm{m}$. Scale bar in "Cornea" panels: $5 \mu \mathrm{m}$. Histological analysis of the negative control (Cas9/tracrRNA ribonuclease complex and no gRNA) is available in Fig. S15

deposition with decreased fibril packing and the loss of collagen layers that also correlated with phenotype severity (Fig. 12O, T, Y). Finally, progressive thinning of the lens epithelium was also observed (Fig. 12I, N, S, X). All these data indicate that cpamd 8 disruption results in early anterior segment maldevelopment, which is characterized mainly by dysgenesis of the iridocorneal angle, the presence of cellular material in the anterior chamber, and disrupted collagen deposition in the corneal stroma.

\section{Discussion}

The primary purpose of this study was to identify by NGS the genetic alterations underlying CG-ASC in sporadic patients with no family history of the disease. This approach led to the identification of several potentially pathogenic CPAMD8 genotypes in four patients. This gene has recently been identified as playing a role in ASD8, an atypical form of ASD, clinically described in three families that, in addition to iris dysgenesis features such as bilateral iris hypoplasia, corectopia, and ectropion uveae, also presented with bilateral lens alterations (i.e., ectopia lentis and cataracts) in the absence of glaucoma and inherited in a recessive fashion, in contrast to typical ASD, which is dominant (Alsaif et al. 2019; Cheong et al. 2016). Our patients also showed recessive inheritance of the disease, though they differ from those of the previous report in terms of the presence of glaucoma and the absence of ectopia lentis but cataracts that could be related to $C P A M D 8$ dysfunction were present in one patient (CG-ASD-ANI-0066). Biallelic CPAMD8 variants have also been found in childhood and juvenile open-angle glaucoma patients (Siggs et al. 2020). Pathogenic CPAMD8 variants were also found in one PCG patient. In addition, the remarkably high frequency of monoallelic rare CPAMD 8 variants (allele frequency $<0.1 \%$ ) in our PCG patients is significantly larger than the frequency of rare SNVs of this gene observed in the gnomAD database v2.1.1 (14\% vs. $1.14 \%$, $p<0.0001$ ) and suggests that they may play a role in nonmonogenic glaucoma, acting as modifier or susceptibility factors, although further studies are required to clarify the involvement of these variants in the disease.

Our findings expand the spectrum of phenotypes associated with this gene, supporting that functional disruption of CPAMD 8 underlies anterior segment alterations ranging from isolated trabeculodysgenesis to varying degrees of iridocorneotrabeculodysgenesis with associated lens abnormalities in some cases. This phenotypic variability can be explained by different variables, including the effects of modifier genes, residual CPAMD8 protein activity, and environmental and/or stochastic factors, and possibly reflects the complexity of ocular development. The pathogenicity of the variants identified in this study is supported by their rarity in the general population and bioinformatic analysis, but further experimental studies are required to firmly establish the deleterious role of some of the missense variants. Interestingly, minigene analysis not only confirmed that one of the identified variants detected in patient CGASD-160 (c.1758+1_1758+4del) altered normal CPAMD8 splicing but also revealed an unexpected pathogenic mechanism for the intronic variant carried by patient PCG-30 (c.4470+10_4470+11insT), related to the identification of a previously uncharacterized alternative transcript. This alternative transcript retains the entire intron 33 and the proximal intron 34 flanking sequence. Intron retention is a mechanism of gene expression control in eukaryotes and is tightly regulated during differentiation and development (Vanichkina et al. 2018). The existence of this alternative mRNA implies that although the insertion of one nucleotide identified in patient PCG-30 does not affect canonical splicing of intron 34, it initiates a frameshift and a PCT in the alternative transcript likely leading to its specific NMD-dependent degradation, with phenotypic manifestations restricted to those tissues in which the corresponding CPAMD8 isoform is expressed. To date, three alternative CPAMD 8 transcripts, translated into three different isoforms (CPAMD8-1a, CPAMD8-1b, and CPAMD8-2), have been described (Cheong et al. 2016; Strausberg et al. 2002). Further studies are required to completely characterize this new identified transcript.

In accordance with our previous studies, the finding of a large proportion (14\%) of CG patients carrying infrequent 


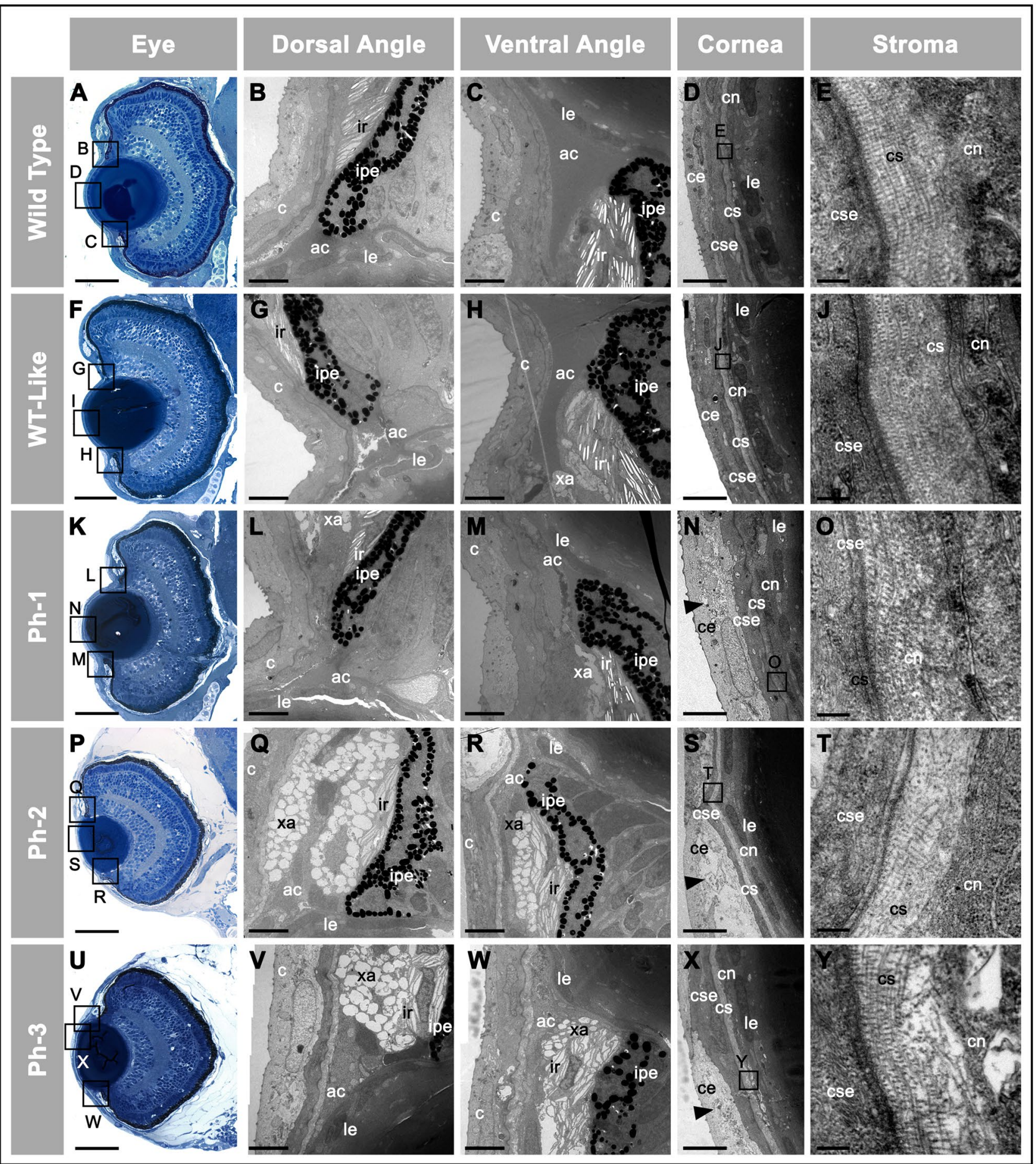

heterozygous $C P A M D 8$ variants also provides evidence for the role of CPAMD8 in non-monogenic or complex congenital glaucoma, reflecting the notions that it participates in the complex genetic regulation of ocular development, and that the combination of genetic defects in more than one of these genes may contribute to the abnormal development of the anterior segment of the eye (Ferre-Fernández et al. 2017; López-Garrido et al. 2013).
Detailed histologic analysis of the anterior chamber angle of one of the patients (CG-ASD-104) with LoF CPAMD8 variants revealed the presence of apparently normal SC and $\mathrm{CC}$, but the abnormal anterior $\mathrm{CM}$ insertion indicates an incomplete and arrested separation of some angle structures. In addition, the remarkable ECM alterations of the $\mathrm{TM}$ are an obvious explanation for the reduced $\mathrm{AH}$ outflow and IOP elevation in this patient and support the notion that 
4Fig. 12 Electron microscopy of F0 zebrafish ocular anterior segment phenotypes resulting from CRISPR/Cas9 cpamd8 inactivation (96-hpf). Thin tissue sections were processed as described in the "Methods" section. A, F, K, P, U Toluidne-blue-stained histological eye sections from Fig. 11 are used as a reference to indicate the position of the electron micrographs. The squares in the "Eye" and "Cornea" panels indicate the areas of the images magnified in the indicated panels. B, C Wild-type dorsal and ventral iridocorneal angles, respectively, showing the early formation of the anterior chamber space and iris structures. D, E Wild-type cornea and corneal stroma, respectively. Note the dense, regularly packed collagen fibrils in the stroma running parallel to each other and that are stacked, forming layers or lamellae. No alterations were observed in the iridocorneal angle $(\mathbf{G}, \mathbf{H})$ or in the cornea $(\mathbf{I}, \mathbf{J})$ of wild type-like phenotypes. $\mathbf{L}$, $\mathbf{Q}, \mathbf{V}$ The primordial dorsal anterior chamber is progressively filled with cellular material, which correlates with phenotype severity from $\mathrm{Ph}-1$ to $\mathrm{Ph}-3 . \mathbf{M}, \mathbf{R}, \mathbf{W}$ A similar alteration is present in the primordial ventral anterior chamber. $\mathbf{N}, \mathbf{S}, \mathbf{X}$ Increasing cytoplasm and organelle swelling (black arrowhead) in the corneal epithelium, suggestive of necrosis. E, J, O, T, Y Progressive collagen disorganization in the corneal stoma correlating with genotype severity, and characterized by decreased packing and irregular deposition of collagen fibrils with loss of collagen lamellae. Squares in different panels indicate enlarged areas. Ir iridophores, $x a$ xantophores, ipe iris pigmented epithelium, $a c$ anterior chamber, $c$ cornea, $c e$ corneal epithelium, $c s e$ corneal subepithelium, $c s$ corneal stroma, $c n$ corneal endothelium, le lens epithelium. Scale bars represent $50 \mu \mathrm{m}$ in the "Eye" panels, $4 \mu \mathrm{m}$ in the dorsal angle, ventral angle and cornea photographs; and $0.2 \mu \mathrm{m}$ in the "stroma" panels

this gene plays a key role in ECM formation in the TM, and likely, in other tissues of the ocular anterior segment. CPAMD8 belongs to the A2M/C3 (alpha-2-macroglobulin/complement 3) protein family (Li et al. 2004), which is composed of large multi-domain proteins with a broad spectrum of endopeptidase inhibitor activity (Rehman et al. 2013). Though the members of this family are evolutionarily distant, their overall architecture is highly conserved and plays multiple functions. A2M, the founding member of this family, is a soluble broad-spectrum proteinase inhibitor (Gonias 1992) that binds and regulates the biological activity of several hormones, cytokines and growth factors (Rehman et al. 2013). Keeping in mind the structural relatedness of CPAMD8 and A2M proteins, we can speculate that CPAMD8 loss-of-function may result in the altered regulation of extracellular proteinases involved in ECM remodeling and/or in the defective regulation of its putative ligands, which might underlie the TM alterations observed in patient CG-ASD-104. Further investigations are required to clarify the precise pathogenic mechanisms underlying CPAMD8associated phenotypes.

Patients with $C Y P 1 B 1$-associated PCG show abnormal CM insertion and ECM alterations in the TM resembling those observed in this study (Garcia-Anton et al. 2017). Trabecular cell death was also a common feature in these two phenotypes, although it is produced by necrosis in CYP1B1defective patients (Zhao et al. 2013) and by apoptosis in CPAMD8-glaucoma. Endothelial trabecular cells maintain the balance between ECM components, such as collagen and elastic fibers (Polansky et al. 1984); therefore, their death may explain (at least in part) the ECM alterations observed in our patient. In addition, the CC and, in some cases the SC, were absent in $C Y P 1 B 1$-deficient patients. Although only one trabeculectomy specimen from patients with $C P A M D 8$ LoF was available, these results indicate that $C Y P 1 B 1$ and $C P A M D 8$ are involved in ECM deposition in the anterior segment and that their independent disruption results in isolated trabeculodysgenesis or broader anterior segment defects.

We also analyze, for the first time, the expression of CPAMD8 in adult human ocular tissues and fluids by Western blot and immunohistochemistry. The presence of a predicted signal peptide in the polypeptide chain of CPAMD8 indicates that this is a secreted protein present extracellularly. In accordance with this idea, western immunoblot revealed proteolytic fragments of this protein in both $\mathrm{AH}$ and vitreous humor. The electrophoretic size of these fragments (approximately 120 and $60-65 \mathrm{kDa}$ ) is consistent with a proteolytic processing of the protein in the RRRR furin cleavage site located at residues 668-671 (Fig. S8A), releasing two fragments with theoretical masses of approximately $133 \mathrm{kDa}$ (N-terminal, 1215-aas) and $71 \mathrm{kDa}$ (C-terminal, 642-aas). The difference between experimental and theoretical molecular masses may be due to posttranslational modifications such as glycosylation and/or to protein conformation. Consistent with this idea, the observed $120-\mathrm{kDa}$ doublet may reflect differences in glycosylation of the C-terminal fragment. Interestingly, the full-length protein (1885 aas and 206.7 kDa predicted molecular mass) was not identified in these fluids indicating that it was completely processed. In line with these results, CPAMD8 fragments derived from posttranslational processing at the RRRR furin cleavage site have also been reported in U251 glioma cells ( $\mathrm{Li}$ et al. 2004). Interestingly, most missense mutations detected in this study and in the previous study (Cheong et al. 2016) are located in the $\mathrm{C}$-terminal fragment of the protein. Based on these data we can speculate that the $\mathrm{C}$-terminal portion of CPAMD8 plays a critical role in the function of the protein. Cleavage of other related proteins such as $\mathrm{C} 3, \mathrm{C} 4$, and $\mathrm{C} 5$, by a furin-type enzyme occurs intracellularly at the tetraarginine linker site as part of the maturation process and results in two chains linked by disulfide bridges (Ricklin et al. 2016). Based on these data, we can propose that the proteolytic processing of CPAMD8 also occurs intracellularly in the secretory pathway, regulating its biological activity, though this hypothesis requires further experimental assessment. The CPAMD8 protein has been identified in the human CB (Goel et al. 2013) and AH (Murthy et al. 2015), while its transcripts have been detected in the NPCE (Janssen et al. 2014). In addition, our results also demonstrate the intracellular presence of CPAMD8 protein in different 
epithelial (NPCE and corneal epithelium), endothelial (corneal endothelium), and muscular cells (ciliary and iris muscles), as well as in fibroblasts from the anterior segment of the eye. The presence of the signal peptide indicates that all these cells synthesize and secrete the protein to their surrounding extracellular medium. In fact, one of the most intense signals was observed in the NPCE, indicating that the protein detected in the AH is secreted, at least partially, by these cells. CPAMD8 was not seen in the adult human TM, which suggests that the protein secreted by the NPCE is transported by the $\mathrm{AH}$ to the iridocorneal angle and to other parts of the ocular anterior segment, where it may play a role in homeostasis and the remodeling of the ECM thanks to its putative activity as a proteinase inhibitor. These ideas are in accordance with our hypothesis of paracrine release of $\mathrm{CB}$ factors capable of affecting the physiology of the TM (CocaPrados and Escribano 2007) as well as with the presence, in the $\mathrm{AH}$, of other proteinase inhibitors, including $\mathrm{A} 2 \mathrm{M}$, secreted by the CB (Coca-Prados et al. 1999; Escribano et al. 1995; Ortego et al. 1997). Altogether, these data indicate not only that CPAMD8 is required for normal anterior segment development but also that it may participate in the homeostasis of this part of the eye, supporting the notion that its functional disruption may underlie the different ASD phenotypes that this study describes. A previous report analyzed the spatiotemporal expression of CPAMD 8 in the developing human eye (9-22 weeks of gestation) by RT-PCR and in situ hybridization, revealing, in accordance with our results, that it is expressed in the lens, iris, cornea, and distal tips of the retinal neuroepithelium that form the iris and CB (Cheong et al. 2016). Similarly, CPAMD8 has been identified in the CB epithelium of healthy fetal and adult cattle (Hollmann et al. 2017).

To gain insight into the role of CPAMD8 in ocular anterior segment development, congenital glaucoma, and ASD, we analyzed the expression of the zebrafish orthologue, cpamd8, in the eyes of early embryos (96 hpf). The protein was detected in mesenchyme-like cells of the anterior segment, indicating that it may participate in the early morphogenesis of the iris, cornea, and lens. CRISPR/Cas9 cpamd8 disruption revealed that $\mathrm{F} 0$ mosaic embryos presented remarkable iridocorneal angle hypoplasia, affecting mainly iris stroma cells, and a primordial anterior chamber filled with abnormal cellular material. Similar anterior chamber alterations have been described in pitx2-deficient zebrafish embryos (72 hpf), probably resulting from the presence of undifferentiated cells, cellular degradation, and/or sloughing and aberrant lipid or protein secretion (Hendee et al. 2018). These similarities suggest that pitx 2 and cpmad 8 might participate in related developmental pathways. Cpamd8disrupted F0 embryos also replicated the corneal thickening observed in two patients (CG-ASD-160 and CG-ASDANI-0066). Interestingly, the abnormal collagen deposition observed in the corneal stroma suggests that cpamd8 is required for normal organization of the ECM structure and resembles collagen disorganization detected in the trabecular meshwork of one of the patients (CG-ASD-104). Moreover, the alteration of the lens epithelium in F0 embryos may correlate with cataracts developed by some ASD8 patients. The phenotypic variability observed in the population of F0 embryos could result from different levels of residual cpamd 8 activity, and the lethal phenotypes might be associated with a complete $\mathrm{LoF}$ of this gene. The establishment of a cpamd 8 knockout zebrafish line will be required for further cpamd8 functional characterization and to determine the role that this gene plays in glaucoma and ASD pathogenesis. Altogether, these results suggest that cpamd8 participates in ocular anterior segment development and in ECM organization, further supporting the notion that its functional disruption underlies a spectrum of CG and ASD phenotypes.

Acknowledgements We are indebted to the patients and their families for cooperation in this study. We would like to thank Ms. MaríaJosé Cabañero for excellent technical assistance and Dr. María-Luisa García-Gil for outstanding electron microscope assistance.

Author contributions J-MB-F and J-DA-A share the first authorship. J-MB-F, J-DA-A, performed the genetic analyses, molecular biology and zebrafish experiments. MC designed the gene panel. MC, CV and AT performed targeted DNA sequencing, ddPCR and data analyses. II contributed with bioinformatic SNV and CNV analysis. M-TG-A, A-IR and J-JS carried out the optical and electron microscopy of human samples. J-MB-F, A-IR and J-JS performed optical and electron microscopy of zebrafish samples. SA-M and RA-A carried out immunohistochemistry and western blot analyses. J-JF-F performed exome sequence analyses. C-DM-H, LM-F, CA, J-MM-d-1-C and JG-F recruited, diagnosed and followed-up patients and supplied human samples. MC-P supplied human tissues and contributed to design expression analyses. J-DA-A and JE supervised the experiments. JE conceived and coordinated the study and wrote the manuscript. All authors contributed to the review and approval of the manuscript.

Funding This study has been supported by research grants from the "Instituto de Salud Carlos III/European Regional Development Fund (ERDF)" (PI15/01193, PI19/00208 and RD16/0008/0019, OFTARED to JE; RD16/0008/0004, OFTARED to JG-F; RD16/0008/0005, OFTARED to A-IR; PI17_01164 to MC and CIBERER 06/07/0036 to CA; https://www.isciii.es), the Ministry of Economy and Competitiveness/ERDF (MINECO, SAF2013-46943-R to MC; https:// www.ciencia.gob.es), the Regional Ministry of Science and Technology of the Board of the Communities of "Castilla-La Mancha" (SBPLY/17/180501/000404 to JE; https://www.educa.jccm.es/idiun iv/es) and the University Chair UAMIIS-FJD of Genomic Medicine, the Ramon Areces Foundation and Regional Government of Madrid (CAM, B2017/BMD3721 to CA). MC was sponsored by the Miguel Servet Program (CPII17_00006) from ISCIII, SA-M was sponsored by the Regional Ministry of Science and Technology of the Board of the Communities of "Castilla-La Mancha" (PREJCCM2016/28) and AT was sponsored by the Regional Government of Madrid/European Regional Development Fund (ERDF) (PEJD-2018-PRE/BMD-9453). M-TG-A was the recipient of a fellowship from the "Ministerio de Educación, Cultura y Deporte" (FPU 13/03308). The funders had no role in study design, data collection and analysis, decision to publish, or preparation of the manuscript. 
Availability of data and material All data are fully available without restriction. Nucleotide sequence data reported have been submitted to GenBank to obtain the corresponding accession numbers.

\section{Compliance with ethical standards}

Conflict of interest The authors have declared that no competing interests exist.

Ethics approval The human study and informed consent procedures were approved by the Ethics Committee for Human Research of the University Hospital Fundación Jiménez Díaz and Hospital Clínico San Carlos (approval number 13/388-E). The research followed the tenets of the Declaration of Helsinki. Informed written consents were obtained prior to participants' inclusion in the study. All animal husbandry and experiments were approved and conducted in accordance with the guidelines set forth by the Institutional Animal Research Committee of the University of Castilla-La Mancha (approval number PR-201504-10).

\section{References}

Ali M, McKibbin M, Booth A, Parry DA, Jain P, Riazuddin SA, Hejtmancik JF, Khan SN, Firasat S, Shires M, Gilmour DF, Towns K, Murphy AL, Azmanov D, Tournev I, Cherninkova S, Jafri H, Raashid Y, Toomes C, Craig J, Mackey DA, Kalaydjieva L, Riazuddin S, Inglehearn CF (2009) Null mutations in LTBP2 cause primary congenital glaucoma. Am J Hum Genet 84:664-671

Alsaif HS, Khan AO, Patel N, Alkuraya H, Hashem M, Abdulwahab F, Ibrahim N, Aldahmesh MA, Alkuraya FS (2019) Congenital glaucoma and CYP1B1: an old story revisited. Hum Genet 138:1043-1049. https://doi.org/10.1007/s00439-018-1878-z

Aroca-Aguilar J-D, Martinez-Redondo F, Martin-Gil A, Pintor J, CocaPrados M, Escribano J (2013) Bicarbonate-dependent secretion and proteolytic processing of recombinant myocilin. PLoS ONE. https://doi.org/10.1371/journal.pone.0054385

Bhattacharya D, Marfo CA, Li D, Lane M, Khokha MK (2015) CRISPR/Cas9: an inexpensive, efficient loss of function tool to screen human disease genes in Xenopus. Dev Biol 408:196-204. https://doi.org/10.1016/j.ydbio.2015.11.003

Campos-Mollo E, Lopez-Garrido M-P, Blanco-Marchite C, GarciaFeijoo J, Peralta J, Belmonte-Martinez J, Ayuso C, Escribano J (2009) CYP1B1 mutations in Spanish patients with primary congenital glaucoma: phenotypic and functional variability. Mol Vis 15:417-431

Ceroni F et al (2019) New GJA8 variants and phenotypes highlight its critical role in a broad spectrum of eye anomalies. Hum Genet 138:1027-1042. https://doi.org/10.1007/s00439-018-1875-2

Chavarria-Soley G, Michels-Rautenstrauss K, Caliebe A, Kautza M, Mardin C, Rautenstrauss B (2006) Novel CYP1B1 and known PAX6 mutations in anterior segment dysgenesis (ASD). J Glaucoma 15:499-504

Cheong SS et al (2016) Mutations in CPAMD8 cause a unique form of autosomal-recessive anterior segment dysgenesis. Am J Hum Genet 99:1338-1352. https://doi.org/10.1016/j.ajhg.2016.09.022

Coca-Prados M, Escribano J (2007) New perspectives in aqueous humor secretion and in glaucoma: the ciliary body as a multifunctional neuroendocrine gland. Prog Retin Eye Res 26:239-262

Coca-Prados M, Escribano J, Ortego J (1999) Differential gene expression in the human ciliary epithelium. Prog Retin Eye Res $18: 403-429$
Colomb E, Kaplan J, Garchon HJ (2003) Novel cytochrome P450 1B1 (CYP1B1) mutations in patients with primary congenital glaucoma in France. Hum Mutat 22:496

Creuzet S, Vincent C, Couly G (2005) Neural crest derivatives in ocular and periocular structures. Int J Dev Biol 49:161-171. https://doi. org/10.1387/ijdb.041937sc

El-Brolosy MA et al (2019) Genetic compensation triggered by mutant mRNA degradation. Nature 568:193-197. https://doi.org/10.1038/ s41586-019-1064-z

Elmore S (2007) Apoptosis: a review of programmed cell death. Toxicol Pathol 35:495-516. https://doi.org/10.1080/019262307013203 37

Escribano J, Ortego J, Coca-Prados M (1995) Isolation and characterization of cell-specific cDNA clones from a subtractive library of the ocular ciliary body of a single normal human donor: transcription and synthesis of plasma proteins. J Biochem (Tokyo) 118:921-931

Ferre-Fernández JJ et al (2017) Whole-exome sequencing of congenital glaucoma patients reveals hypermorphic variants in GPATCH3, a new gene involved in ocular and craniofacial development. Sci Rep 7:46175. https://doi.org/10.1038/srep46175

Garcia-Anton MT et al (2017) Goniodysgenesis variability and activity of CYP1B1 genotypes in primary congenital glaucoma. PLoS ONE. https://doi.org/10.1371/journal.pone.0176386

Goel R et al (2013) Characterizing the normal proteome of human ciliary body. Clin Proteomics 10:9. https://doi. org/10.1186/1559-0275-10-9

Gonias SL (1992) Alpha 2-macroglobulin: a protein at the interface of fibrinolysis and cellular growth regulation. Exp Hematol 20:302-311

Gould DB, John SW (2002) Anterior segment dysgenesis and the developmental glaucomas are complex traits. Hum Mol Genet 11:1185-1193

Gould DB, Smith RS, John SW (2004) Anterior segment development relevant to glaucoma. Int J Dev Biol 48:1015-1029

Hanson IM et al (1994) Mutations at the PAX6 locus are found in heterogeneous anterior segment malformations including Peters' anomaly. Nat Genet 6:168-173. https://doi.org/10.1038/ng029 4-168

Hendee KE et al (2018) PITX2 deficiency and associated human disease: insights from the zebrafish model. Hum Mol Genet 27:16751695. https://doi.org/10.1093/hmg/ddy074

Hollmann AK et al (2017) Morgagnian cataract resulting from a naturally occurring nonsense mutation elucidates a role of CPAMD8 in mammalian lens development. PLoS ONE 12:e0180665. https ://doi.org/10.1371/journal.pone.0180665

Janssen SF, Gorgels TG, Ten Brink JB, Jansonius NM, Bergen AA (2014) Gene expression-based comparison of the human secretory neuroepithelia of the brain choroid plexus and the ocular ciliary body: potential implications for glaucoma. Fluids Barriers CNS 11:2. https://doi.org/10.1186/2045-8118-11-2

Jordan T et al (1992) The human PAX6 gene is mutated in two patients with aniridia. Nat Genet 1:328-332. https://doi.org/10.1038/ ng0892-328

Kaur K et al (2005) Myocilin gene implicated in primary congenital glaucoma. Clin Genet 67:335-340

Kupfer C, Kaiser-Kupfer MI (1978) New hypothesis of developmental anomalies of the anterior chamber associated with glaucoma. Trans Ophthalmol Soc UK 98:213-215

Kupfer C, Kaiser-Kupfer MI (1979) Observations on the development of the anterior chamber angle with reference to the pathogenesis of congenital glaucomas. Am J Ophthalmol 88:424-426

Li ZF, Wu XH, Engvall E (2004) Identification and characterization of CPAMD8, a novel member of the complement 3/alpha2-macroglobulin family with a C-terminal Kazal domain. Genomics 83:1083-1093. https://doi.org/10.1016/j.ygeno.2003.12.005 
Livak KJ, Schmittgen TD (2001) Analysis of relative gene expression data using real-time quantitative PCR and the 2(-Delta Delta C(T)) Method. Methods 25:402-408. https://doi.org/10.1006/ meth.2001.1262

López-Garrido M-P, Medina-Trillo C, Morales-Fernandez L, GarciaFeijoo J, Martínez-De-La-Casa J-M, García-Antón M, Escribano J (2013) Null CYP1B1 genotypes in primary congenital and nondominant juvenile glaucoma. Ophthalmology 120:716-723. https ://doi.org/10.1016/j.ophtha.2012.09.016

Medina-Trillo C et al (2016) Rare FOXC1 variants in congenital glaucoma: identification of translation regulatory sequences. Eur J Hum Genet 24:672-680. https://doi.org/10.1038/ejhg.2015.169

Medina-Trillo C et al (2015) Hypo- and hypermorphic FOXC1 mutations in dominant glaucoma: transactivation and phenotypic variability. PLoS ONE 10:e0119272. https://doi.org/10.1371/journ al.pone. 0119272

Murthy KR et al (2015) Proteomics of human aqueous humor. OMICS 19:283-293. https://doi.org/10.1089/omi.2015.0029

Nishimura DY et al (1998) The forkhead transcription factor gene FKHL7 is responsible for glaucoma phenotypes which map to 6p25. Nat Genet 19:140-147

Ortego J, Escribano J, Coca-Prados M (1997) Gene expression of proteases and protease inhibitors in the human ciliary epithelium and ODM-2 cells. Exp Eye Res 65:289-299

Pagani F et al (2003) New type of disease causing mutations: the example of the composite exonic regulatory elements of splicing in CFTR exon 12. Hum Mol Genet 12:1111-1120. https://doi. org/10.1093/hmg/ddg131

Papadopoulos M et al (2013) Establishing the diagnosis and determining glaucoma progression. In: Weinreb R, Grajewski A, Papadopoulos M, Grigg J, Freedman S (eds) Childhood glaucoma. Kluger Publications, Amsterdam, pp 15-41

Polansky JR, Wood IS, Maglio MT, Alvarado JA (1984) Trabecular meshwork cell culture in glaucoma research: evaluation of biological activity and structural properties of human trabecular cells in vitro. Ophthalmology 91:580-595. https://doi.org/10.1016/ s0161-6420(84)34241-5

Rehman AA, Ahsan H, Khan FH (2013) $\alpha$-2-Macroglobulin: a physiological guardian. J Cell Physiol 228:1665-1675. https://doi. org/10.1002/jcp.24266

Reis LM, Semina EV (2011) Genetics of anterior segment dysgenesis disorders. Curr Opin Ophthalmol 22:314-324. https://doi. org/10.1097/ICU.0b013e328349412b

Ricklin D, Reis ES, Mastellos DC, Gros P, Lambris JD (2016) Complement component C3: the "Swiss Army Knife" of innate immunity and host defense. Immunol Rev 274:33-58. https://doi. org/10.1111/imr.12500
Sarfarazi M, Stoilov I (2000) Molecular genetics of primary congenital glaucoma. Eye 14(Pt 3B):422-428

Semina EV et al (1996) Cloning and characterization of a novel bicoidrelated homeobox transcription factor gene, RIEG, involved in Rieger syndrome. Nat Genet 14:392-399

Siggs OM et al (2020) Biallelic CPAMD8 variants are a frequent cause of childhood and Juvenile open-angle glaucoma. Ophthalmology. https://doi.org/10.1016/j.ophtha.2019.12.024

Souma T et al (2016) Angiopoietin receptor TEK mutations underlie primary congenital glaucoma with variable expressivity. J Clin Invest 126:2575-2587. https://doi.org/10.1172/JCI85830

Sowden JC (2007) Molecular and developmental mechanisms of anterior segment dysgenesis. Eye (Lond) 21:1310-1318

Stoilov I, Akarsu AN, Sarfarazi M (1997) Identification of three different truncating mutations in cytochrome P4501B1 (CYP1B1) as the principal cause of primary congenital glaucoma (Buphthalmos) in families linked to the GLC3A locus on chromosome 2p21. Hum Mol Genet 6:641-647

Strausberg RL et al (2002) Generation and initial analysis of more than 15,000 full-length human and mouse cDNA sequences. Proc Natl Acad Sci USA 99:16899-16903. https://doi.org/10.1073/ pnas. 242603899

Vanichkina DP, Schmitz U, Wong JJ, Rasko JEJ (2018) Challenges in defining the role of intron retention in normal biology and disease. Semin Cell Dev Biol 75:40-49. https://doi.org/10.1016/j.semcd b.2017.07.030

Vincent A et al (2006) Further support of the role of CYP1B1 in patients with Peters anomaly. Mol Vis 12:506-510

Vincent A et al (2001) Phenotypic heterogeneity of CYP1B1: mutations in a patient with Peters' anomaly. J Med Genet 38:324-326

Weisschuh N, Wolf C, Wissinger B, Gramer E (2009) A clinical and molecular genetic study of German patients with primary congenital glaucoma. Am J Ophthalmol 147:744-753

Westerfield M (2000) The Zebrafish book: a guide for the laboratory use of Zebrafish (Danio rerio), 5th edn. University of Oregon Press, Eugene

Zhao Y et al (2013) Cyp1b1 mediates periostin regulation of trabecular meshwork development by suppression of oxidative stress. Mol Cell Biol 33:4225-4240. https://doi.org/10.1128/MCB.00856-13

Publisher's Note Springer Nature remains neutral with regard to jurisdictional claims in published maps and institutional affiliations.

\section{Affiliations}

\section{Juan-Manuel Bonet-Fernández ${ }^{1,2,3} \cdot$ José-Daniel Aroca-Aguilar ${ }^{1,2,3} \cdot$ Marta Corton $^{4,5}$. Ana-Isabel Ramírez ${ }^{3,6}$. Susana Alexandre-Moreno ${ }^{1,2,3} \cdot$ María-Teresa García-Antón $^{6}$. Juan-José Salazar³,6 • Jesús-José Ferre-Fernández ${ }^{1,2,3}$. Raquel Atienzar-Aroca ${ }^{1,2,3}$. Cristina Villaverde ${ }^{4,5}$. Ionut lancu ${ }^{4,5}$. Alejandra Tamayo ${ }^{4}$. Carmen-Dora Méndez-Hernández $z^{3,7,8}$. Laura Morales-Fernández ${ }^{3,7,8}$. Blanca Rojas ${ }^{3,6,9}$. Carmen Ayuso $0^{4,5}$. Miguel Coca-Prados ${ }^{10}$. José-Maria Martinez-de-la-Casa ${ }^{3,7,8}$. Julián García-Feijoo ${ }^{3,7,8}$ • Julio Escribano 1,2,3 (1)}

1 Área de Genética, Facultad de Medicina de Albacete, Universidad de Castilla-La Mancha, Albacete, Spain

2 Instituto de Investigación en Discapacidades Neurológicas (IDINE), Universidad de Castilla-La Mancha, Albacete, Spain
3 Cooperative Research Network on Age-Related Ocular Pathology, Visual and Life Quality (OFTARED), Instituto de Salud Carlos III, Madrid, Spain

4 Área de Genética and Genómica, Instituto de Investigación Sanitaria-Hospital Universitario Fundación Jiménez Díaz-Universidad Autónoma de Madrid (IIS-FJD, UAM), Madrid, Spain 
5 Centro de Investigación Biomédica en Red de Enfermedades Raras (CIBERER), Madrid, Spain

6 Instituto de Investigaciones Oftalmológicas Ramón Castroviejo, Universidad Complutense de Madrid, Madrid, Spain

7 Servicio de Oftalmología, Hospital San Carlos, Madrid, Spain
8 Instituto de Investigación Sanitaria del Hospital Clínico San Carlos, Madrid, Spain

9 Facultad de Medicina, Universidad Complutense de Madrid, Madrid, Spain

10 Department of Ophthalmology and Visual Science, Yale University School of Medicine, New Haven, CT, USA 